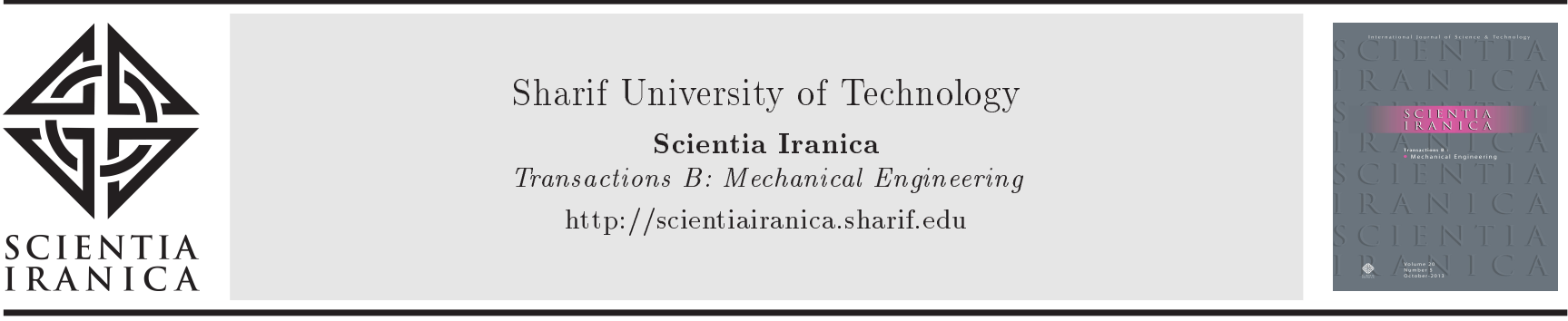

\title{
Novel aspects of Soret and Dufour in entropy generation minimization for Williamson fluid flow
}

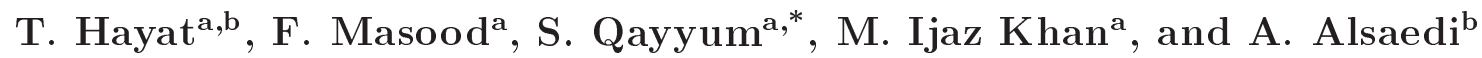 \\ a. Department of Mathematics, Quaid-I-Azam University 45320, Islamabad 44000, Pakistan. \\ b. Nonlinear Analysis and Applied Mathematics (NAAM) Research Group, Department of Mathematics, Faculty of Science, King \\ Abdulaziz University, Jeddah 21589, Saudi Arabia.
}

Received 27 December 2018; received in revised form 4 February 2019; accepted 27 April 2019

\author{
KEYWORDS \\ Williamson fluid; \\ Rotating disks; \\ Activation energy; \\ Viscous dissipation; \\ Entropy generation; \\ Soret and Dufour \\ effects; \\ Stratification.
}

\begin{abstract}
Soret and Dufour effects on MHD flow of Williamson fluid between two rotating disks are examined in this study. Impacts of stratification, viscous dissipation, and activation energy are also considered. Bejan number and entropy generation for the stratified flow are discussed. The governing Partial Differential Equations (PDEs) are converted into ODE using von Kármán transformations. The convergent solution of complicated ODE is found using a homotopic procedure. The results of physical quantities are discussed through plots and numerical values. It is noted that axial and radial velocities are higher in the case of greater Weissenberg number. Temperature and concentration profiles are the decreasing functions of thermal and solutal stratification parameters, respectively. Entropy and Bejan number show the opposite trends for higher Weissenberg number and Brinkman number.
\end{abstract}

(C) 2020 Sharif University of Technology. All rights reserved.

\section{Introduction}

Recent researchers have particularly focused on the flows of non-Newtonian fluids because these liquids have penetrated industrial and technological processes. In this regard, they have suggested a number of models for such liquids due to their diverse properties. In general, subclasses of differential-type liquids, known as second, third, and fourth grades, are extensively analyzed. However, Williamson fluid model has received insignificant attention. Williamson [1] studied the pseudoplastic material experimentally. Nadeem et al. [2] discussed Williamson fluid flow that results from the stretching velocity of a surface. This flow, which is characterized by pressure-dependent viscosity,

\footnotetext{
*. Corresponding author.

E-mail address: sumaira@math.qau.edu.pk (S. Qayyum)
}

doi: $10.24200 /$ sci. 2019.52553 .2772 was numerically analyzed by Zehra et al. [3]. Khan et al. [4] and Qayyum et al. [5] studied entropy generation during heat transfer of Williamson nanofluid due to stretching sheet and rotating disks, respectively. Further studies on non-Newtonian fluids can be seen in $[6-10]$.

Non-Newtonian fluid flow with rotating disks has many applications in engineering, e.g., air cleaning machine, medical equipment and aerodynamical engineering processes, etc. Karman [11] firstly examined the steady flow using an infinite rotating disk. The flow generated by rotating disks with radiative heat flux and variable thickness was examined by Hayat et al. [12]. Khan et al. [13] presented MHD EyringPowell fluid flow with a rotating disk. Doh and Muthtamilselvan discussed Micropolar fluid flow by a rotating disk with MHD and thermophoretic particle deposition effects [14]. Khan et al. examined magnetic field and double diffusion in the couple stress fluid flow by a rotating disk [15]. Griffiths et al. addressed the 
stability analysis of flow using a rotating disk [16]. Qayyum et al. [17] discussed disorder in the system based on Williamson fluid motion.

It is a well-known fact that mass transfer exists in view of species concentration difference in a mixture. The species characterized by different concentrations transport themselves in a mixture from a region with higher concentration to a region with lower concentration. Moreover, activation energy is the minimum quantity of energy that must be possessed by reactants before any specified chemical reaction occurs. This process, which is followed by a chemical reaction with activation energy in mass transfer, is usually used in food processing, geothermal reservoirs, chemical engineering, etc. Bestman investigated convection of a binary mixture flowing in a porous space with activation energy [18]. Makinde et al. discussed unsteady radiative flow in the presence of chemical reaction [19]. Awad et al. described unsteady rotating flow with activation energy and chemical reaction [20]. Shafique et al. studied the rotating flow containing chemically reactive species and activation energy [21].

In recent years, to find optimal designs for engineering system, the second law of thermodynamics has been applied to the analysis of Entropy Generation Minimization (EGM). EGM helps determine the growing rate of irreversibility during a process. Reversibility of heat and mass transfer and irreversibility of viscous dissipation can be determined by entropy generation. During the convection process, irreversibility takes place inside the cavity. It is quite necessary to diminish the irreversibility process to ensure energy conservation. To study the effects of entropy generation inside a thermal system, the second law of thermodynamics is considered. Firstly, Bejan introduced entropy generation [22] and explained that entropy was generated due to viscous effects and thermal conductivity. Amani and Nobari investigated entropy generation in a curved pipe at a constant wall temperature [23]. Hayat et al. studied peristaltic rotating flow of nanoparticles with entropy generation [24]. Shit et al. found that nanofluid flow resulted from exponential stretching sheet and entropy generation [25]. Some of the recent works about entropy generation can be seen in [26-30].

Here, this study analyzes the effects of entropy generation on MHD Williamson fluid flow between two rotating disks. Further stratification, viscous dissipation, and activation energy effects are considered. To the best of the authors' knowledge, such an attempt at studying Williamson fluid has not been considered yet. Homotopy technique [31-42] is used to develop convergent solutions. Behaviors of temperature, velocity, entropy generation, Nusselt number, Bejan number, and skin friction are discussed via graphs and tabulated values. At the end, concluding remarks are given.

\section{Formulation}

Three-dimensional steady flow of Williamson fluid between two rotating stretchable disks was considered. This study aims to scrutinize FGM in flow with effects of Dufour/Soret, stratification, and viscous dissipation. Chemical reaction with activation energy was also investigated. Flow takes place due to the stretching of disks. The lower $(z=0)$ and upper $(z=h)$ disks are characterized by respective angular velocities of $\Omega_{1}$ and $\Omega_{2}$. Flow is caused by the stretching of lower disk. Lower and upper disks correspond to temperatures $\left(\hat{T}_{1}, \hat{T}_{2}\right)$ and concentration $\left(\hat{C}_{1}, \hat{C}_{2}\right)$. A uniform magnetic field of strength $\left(B_{0}\right)$ is exerted in the $Z$-direction (see Figure 1).

An extra stress tensor $(\bar{\tau})$ of Williamson fluid is given below:

$$
\bar{\tau}=\left[\tilde{\mu}_{\infty}+\frac{\left(\tilde{\mu}_{0}-\tilde{\mu}_{\infty}\right)}{1-\Gamma \dot{\gamma}}\right] \mathbf{A},
$$

where $\mathbf{A}$ denotes the first Rilvin-Erickson tensor, $\tilde{\mu}_{0}$ is the zero shear rate viscosity, $\tilde{\mu}_{\infty}$ is the infinite shear rate viscosity, and $\Gamma>0$ is a time constant. Herein, $\dot{\gamma}$ is defined below:

$$
\dot{\gamma}=\sqrt{\frac{1}{2}\left(\operatorname{tr} \mathbf{A}^{2}\right)} \text {. }
$$

Through Eq. (1), one obtains the following:

$$
\bar{\tau}=\left(\tilde{\mu}_{0}+\left(\tilde{\mu}_{0}-\tilde{\mu}_{\infty}\right) \Gamma \dot{\gamma}\right) \mathbf{A},
$$

which further yields:

$$
\bar{\tau}_{r r}=2\left(\left(\tilde{\mu}_{0}-\tilde{\mu}_{\infty}\right) \Gamma \dot{\gamma}+\tilde{\mu}_{0}\right)\left(\frac{\partial \hat{u}}{\partial r}\right),
$$

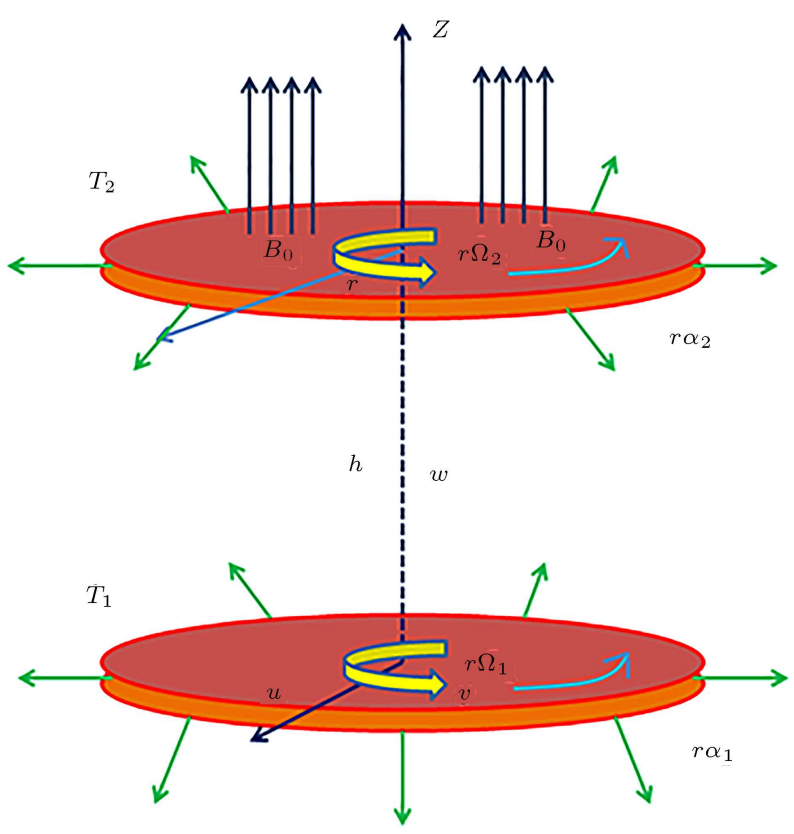

Figure 1. Schematic diagram of the problem. 


$$
\begin{aligned}
& \bar{\tau}_{\theta \theta}=2\left(\left(\tilde{\mu}_{0}-\tilde{\mu}_{\infty}\right) \Gamma \dot{\gamma}+\tilde{\mu}_{0}\right)\left(\frac{1}{r} \frac{\partial \hat{v}}{\partial \theta}+\frac{\hat{u}}{r}\right), \\
& \bar{\tau}_{z z}=2\left(\left(\tilde{\mu}_{0}-\tilde{\mu}_{\infty}\right) \Gamma \dot{\gamma}+\tilde{\mu}_{0}\right)\left(\frac{\partial \hat{w}}{\partial z}\right), \\
& \bar{\tau}_{\theta r}=\bar{\tau}_{r \theta}=\left(\left(\tilde{\mu}_{0}-\tilde{\mu}_{\infty}\right) \Gamma \dot{\gamma}+\tilde{\mu}_{0}\right)\left(\frac{1}{r} \frac{\partial \hat{u}}{\partial \theta}+\frac{\partial \hat{v}}{\partial r}-\frac{\hat{v}}{r}\right), \\
& \bar{\tau}_{r z}=\bar{\tau}_{z r}=\left(\left(\tilde{\mu}_{0}-\tilde{\mu}_{\infty}\right) \Gamma \dot{\gamma}+\tilde{\mu}_{0}\right)\left(\frac{\partial \hat{w}}{\partial r}+\frac{\partial \hat{u}}{\partial z}\right), \\
& \bar{\tau}_{z \theta}=\bar{\tau}_{\theta z}=\left(\left(\tilde{\mu}_{0}-\tilde{\mu}_{\infty}\right) \Gamma \dot{\gamma}+\tilde{\mu}_{0}\right)\left(\frac{1}{r} \frac{\partial \hat{w}}{\partial \theta}+\frac{\partial \hat{v}}{\partial z}\right) .
\end{aligned}
$$

Eq. (5) is shown in Box I. Mathematical statements of the problem under consideration satisfy the following $[5]$ :

$$
\begin{aligned}
& \frac{\partial \hat{u}}{\partial r}+\frac{\hat{u}}{r}+\frac{\partial \hat{w}}{\partial z}=0, \\
& \rho\left(w \frac{\partial \hat{u}}{\partial z}+\hat{u} \frac{\partial \hat{u}}{\partial r}-\frac{\hat{v}^{2}}{r}\right) \\
& =-\frac{\partial p}{\partial r}+\frac{\partial \bar{\tau}_{r r}}{\partial r}+\frac{\partial \bar{\tau}_{z r}}{\partial z}+\frac{\bar{\tau}_{r r}-\bar{\tau}_{\theta \theta}}{r}-\sigma B_{0}^{2} \hat{u}, \\
& \rho\left(\hat{u} \frac{\partial \hat{v}}{\partial r}+w \frac{\partial \hat{v}}{\partial z}+\frac{\hat{v} \hat{u}}{r}\right) \\
& =\frac{\partial \bar{\tau}_{r \theta}}{\partial r}+\frac{\partial \bar{\tau}_{z \theta}}{\partial z}+2 \frac{\bar{\tau}_{r \theta}}{r}-\sigma B_{0}^{2} \hat{v}, \\
& \rho\left(\hat{u} \frac{\partial w}{\partial r}+w \frac{\partial w}{\partial z}\right)=-\frac{\partial p}{\partial z}+\frac{\partial \bar{\tau}_{r z}}{\partial r}+\frac{\partial \bar{\tau}_{z z}}{\partial z}+\frac{\bar{\tau}_{r z}}{r}, \\
& {\left[\hat{u} \frac{\partial \hat{T}}{\partial r}+\hat{w} \frac{\partial \hat{T}}{\partial z}\right]=\frac{k}{\rho C_{p}}\left(\frac{\partial^{2} \hat{T}}{\partial z^{2}}+\frac{\partial^{2} \hat{T}}{\partial r^{2}}+\frac{1}{r} \frac{\partial \hat{T}}{\partial r}\right)} \\
& +\bar{\tau} \cdot L+\frac{D_{m} K_{T}}{C_{p} C_{s}}\left(\frac{\partial^{2} \hat{C}}{\partial r^{2}}+\frac{\partial^{2} \hat{C}}{\partial z^{2}}+\frac{1}{r} \frac{\partial \hat{C}}{\partial r}\right), \\
& \hat{u} \frac{\partial \hat{C}}{\partial r}+\hat{w} \frac{\partial \hat{C}}{\partial z}=D\left(\frac{\partial^{2} \hat{C}}{\partial r^{2}}+\frac{\partial^{2} \hat{C}}{\partial z^{2}}+\frac{1}{r} \frac{\partial \hat{C}}{\partial r}\right) \\
& +\frac{D_{m} K_{T}}{\hat{T}_{m}}\left(\frac{1}{r} \frac{\partial \hat{T}}{\partial r}+\frac{\partial^{2} \hat{T}}{\partial r^{2}}+\frac{\partial^{2} \hat{T}}{\partial z^{2}}\right)
\end{aligned}
$$

$$
\dot{\gamma}=\sqrt{2\left[\left(\frac{\partial \hat{u}}{\partial r}\right)^{2}+\left(\frac{\partial \hat{w}}{\partial z}\right)^{2}+\left(\frac{\hat{u}}{r}+\frac{1}{r} \frac{\partial \hat{v}}{\partial \theta}\right)^{2}\right]+\left(\frac{1}{r} \frac{\partial \hat{u}}{\partial \theta}+\frac{\partial \hat{v}}{\partial r}-\frac{\hat{v}}{r}\right)^{2}+\left(\frac{\partial \hat{v}}{\partial z}+\frac{1}{r} \frac{\partial \hat{w}}{\partial \theta}\right)^{2}+\left(\frac{\partial \hat{w}}{\partial r}+\frac{\partial \hat{u}}{\partial z}\right)^{2}}
$$

$$
\begin{aligned}
& \hat{u}=a_{1} r, \quad \hat{v}=\Omega_{1} r, \quad \hat{w}=0, \\
& \hat{T}=\hat{T}_{1}=m_{1} r+\hat{T}_{0}, \quad \hat{C}=\hat{C}_{1}=m_{3} r+\hat{C}_{0}, \\
& \text { at } z=0, \quad \hat{u}=a_{2} r, \quad \hat{v}=\Omega_{2} r, \quad p=0, \\
& \hat{C}=\hat{C}_{2}=m_{4} r+\hat{C}_{0}, \quad \hat{T}=\hat{T}_{2}=m_{2} r+\hat{T}_{0} \\
& \text { at } z=h,
\end{aligned}
$$

where, in $(x, y, z)$ directions, the velocity components are $(\breve{u}, \breve{v}, \hat{w})$, respectively, $p$ the hydrostatic pressure, $C_{p}$ specific heat, $\rho$ density of fluid, $k$ thermal conductivity, $D_{m}$ the effective diffusivity rate of mass, $C_{s}$ the susceptibility of concentration, $K_{T}$ thermal-diffusion ratio, $k_{r}$ the reaction rate, $\sigma$ electrical conductivity, $E_{\alpha}$ activation energy where $\left(\kappa=8.61 \times 10^{-5} \mathrm{eV} / \mathrm{K}\right)$ the Boltzmann constant, $n$ the fitted rate constant, and $T_{m}$ mean temperature of fluid.

Suitable transformations for this analysis are given as follows:

$$
\begin{aligned}
& \hat{u}=r \Omega_{1} \hat{f}(\xi), \quad \hat{v}=r \Omega_{1} \hat{g}(\xi), \quad \hat{w}=h \Omega_{1} \hat{h}(\xi), \\
& \hat{\varphi}(\xi)=\frac{\hat{C}-\hat{C}_{2}}{\hat{C}_{1}-\hat{C}_{0}}, \quad \hat{P}(\xi)=\frac{\hat{p}}{\rho h^{2} \Omega_{1}^{2}}, \\
& \hat{\theta}(\xi)=\frac{\hat{T}-\hat{T}_{2}}{\hat{T}_{1}-\hat{T}_{0}}, \quad \xi=\frac{z}{h}
\end{aligned}
$$

Eq. (1) is satisfied and Eqs. (7)-(13) take the following forms:

$$
\hat{h}^{\prime}+2 \hat{f}=0,
$$




$$
\begin{aligned}
&(1+\mathrm{We} \dot{\gamma}) \hat{f}^{\prime \prime}+\operatorname{Re}\left(\hat{g}^{2}-\hat{f}^{2}-M \hat{f}-\hat{h} \hat{f}^{\prime}\right) \\
&+\frac{\mathrm{We} A^{2}}{\dot{\gamma}}\left(\hat{f}^{\prime \prime} \hat{f}^{\prime 2}+\hat{g}^{\prime \prime} \hat{g}^{\prime} \hat{f}^{\prime}\right) \\
&+\frac{2 \mathrm{We}}{\dot{\gamma}}\left(\hat{h}^{\prime \prime} \hat{h}^{\prime} \hat{f}^{\prime}+3 \hat{f}^{\prime 2} \hat{f}+\hat{g}^{2} \hat{f}\right)=0, \\
&(1+\mathrm{We} \dot{\gamma}) \hat{g}^{\prime \prime}+\frac{\mathrm{WeRe}}{\dot{\gamma}}\left(\hat{g}^{\prime \prime} \hat{g}^{2}+\hat{g}^{\prime \prime} \hat{g}^{\prime} \hat{f}^{\prime}\right) \\
& \quad-\left(\hat{h} \hat{g}^{\prime}+2 \hat{g} \hat{f}+M \hat{g}\right)+\frac{2 \mathrm{We}}{\dot{\gamma}}\left(\hat{g}^{\prime} \hat{h}^{\prime} \hat{h}^{\prime \prime}+2 \hat{g}^{\prime} \hat{f} \hat{f}^{\prime}\right) \\
& \quad=0,
\end{aligned}
$$

$$
\begin{aligned}
\operatorname{Re}\left(P^{\prime}\right. & \left.+\hat{h}^{\prime} \hat{h}\right)-\frac{\mathrm{We} A^{2}}{\dot{\gamma}}\left(\hat{f}^{\prime 3}+\hat{f}^{\prime} \hat{g}^{\prime 2}+\hat{f}^{\prime \prime} \hat{f}^{\prime} \hat{h}^{\prime}+\hat{g}^{\prime \prime} \hat{g}^{\prime} \hat{h}^{\prime}\right) \\
& -2(1+\mathrm{We} \dot{\gamma})\left(\hat{f}^{\prime}+\hat{h}^{\prime \prime}\right) \\
& -\frac{4 \mathrm{We}}{\dot{\gamma}}\left(2 \hat{f} \hat{f}^{\prime} \hat{h}^{\prime}+\hat{h}^{\prime \prime} \hat{h}^{\prime 2}\right)=0,
\end{aligned}
$$

$$
\begin{aligned}
\operatorname{Re} \hat{\theta}^{\prime \prime} & +\left(\hat{\theta}+S_{1}\right)-(\operatorname{Re})(\operatorname{Pr})\left(\hat{h} \hat{\theta}^{\prime}+S_{1} \hat{f}+\hat{\theta} \hat{f}\right) \\
& +D_{f}\left(\operatorname{Re} \hat{\varphi}^{\prime \prime}+\hat{\varphi}+S_{2}\right)(\operatorname{Ec})(\operatorname{Pr})(1+W e \dot{\gamma}) \\
& \left(4 \hat{f}^{2}+\operatorname{Re} \hat{f}^{\prime 2}+\operatorname{Re} \hat{g}^{\prime 2}+2 \hat{h}^{\prime 2}\right)=0
\end{aligned}
$$

$$
\begin{aligned}
\operatorname{Re} \hat{\varphi}^{\prime \prime} & -\operatorname{ReSc} \hat{f}\left(S_{2}+\hat{\varphi}\right)-\operatorname{Re}(\operatorname{Sc}) \hat{\varphi}^{\prime} \hat{h} \\
& +S_{r}\left(\operatorname{Re} \hat{\theta}^{\prime \prime}+S_{1}+\hat{\theta}\right)+\left(\hat{\varphi}+S_{2}\right) \\
& -\sigma(\operatorname{Re}) \operatorname{Sc}(1+\delta \hat{\theta})^{n} \exp \left(\frac{-E}{1+\delta \hat{\theta}}\right) \hat{\varphi}=0,
\end{aligned}
$$

with boundary conditions:

$$
\begin{aligned}
& \hat{f}(0)=A_{1}, \quad \hat{h}(0)=0, \quad \hat{g}(0)=1, \\
& \hat{\varphi}(0)=1-S_{2}, \quad \hat{\theta}(0)=1-S_{1}, \quad \text { at } \quad \xi=0, \\
& \hat{f}(1)=A_{2}, \quad \hat{g}(1)=\Omega, \quad \hat{\theta}(1)=0, \quad P(1)=0, \\
& \hat{\varphi}(1)=0, \quad \text { at } \quad \xi=1 . \\
& \dot{\gamma}=\sqrt{4 \hat{f}^{2}+A^{2}\left(\hat{f}^{\prime 2}+\hat{g}^{\prime 2}\right)+2 \hat{h}^{\prime 2}}
\end{aligned}
$$

where:

$$
\begin{aligned}
& M=\frac{\sigma B_{0}^{2}}{\rho \Omega_{1}}, \quad \operatorname{Re}=\frac{\Omega_{1} r^{2}}{\nu}, \quad \sigma=\frac{k_{r}^{2}}{\Omega_{1}}, \\
& E=\frac{E_{\alpha}}{\kappa \hat{T}_{2}}, \quad S_{r}=\frac{D_{m} K_{T}}{D \bar{T}_{m}} \frac{\hat{T}_{1}-\hat{T}_{0}}{\hat{C}_{1}-\hat{C}_{0}}, \quad \delta=\frac{\hat{T}_{1}-\hat{T}_{0}}{\hat{T}_{2}},
\end{aligned}
$$

$$
\begin{aligned}
& \operatorname{Pr}=\frac{\mu_{0} c_{p}}{k}, \quad S_{2}=\frac{\hat{C}_{2}-\hat{C}_{0}}{\hat{C}_{1}-\hat{C}_{0}}, \quad D_{f}=\frac{\rho D_{m} K_{T}}{k C_{s}} \\
& \frac{\hat{C}_{1}-\hat{C}_{0}}{\hat{T}_{1}-\hat{T}_{0}}, \quad A_{1}=\frac{a_{1}}{\Omega_{1}}, \quad A_{2}=\frac{a_{2}}{\Omega_{1}}, \\
& \mathrm{We}=\frac{\Omega \Gamma\left(\mu_{0}-\mu_{\infty}\right)}{\mu_{0}}, \quad \mathrm{Sc}=\frac{\nu}{D}, \quad S_{1}=\frac{\hat{T}_{2}-\hat{T}_{0}}{\hat{T}_{1}-\hat{T}_{0}}, \\
& \mathrm{Ec}=\frac{\Omega^{2} r^{2}}{c_{p}\left(\hat{T}_{1}-\hat{T}_{0}\right)}, \quad A^{2}=\frac{r^{2}}{h^{2}} .
\end{aligned}
$$

Herein, Re represents the local Reynold number, Ec Eckert number, Pr Prandtl number, We Weissenberg number, $M$ magnetic field parameter, Sc Schmidt number, $D_{f}$ Dufour number, Sr Soret number, $S_{1}$ thermal stratification parameter, $S_{2}$ solutal stratification parameter, $E$ dimensionless activation energy, $\sigma$ dimensionless reaction rate, and $\delta$ temperature difference parameter.

Radial and tangential shear stresses and heat transfer rate at the lower disk are given below:

$$
\begin{aligned}
C_{\hat{f}_{r 1}} & =\frac{\left.\tau_{r z}\right|_{z=0}}{\mu_{0} \Omega_{1}}, \quad C_{\breve{f}_{\hat{\theta} 1}}=\frac{\left.\tau_{\theta z}\right|_{z=0}}{\mu_{0} \Omega_{1}}, \\
\mathrm{Nu}_{r 1} & =\frac{\left.r q_{w}\right|_{z=0}}{k\left(\hat{T}_{1}-\hat{T}_{0}\right)}
\end{aligned}
$$

where $q_{w}$ is:

$$
q_{w}=-k \frac{\partial \hat{T}}{\partial z}
$$

The definitions of Nusselt number and skin friction coefficients at the lower disk are:

$$
\begin{aligned}
\mathrm{Nu}_{r 1}= & -\sqrt{\operatorname{Re}} \hat{\theta}^{\prime}(0), \\
\frac{C_{\hat{f}_{r 1}}}{A}= & {\left[1+(\mathrm{We}) \hat{f}^{\prime}(0)\right.} \\
& \sqrt{\left.4 \hat{f}^{2}(0)+A^{2}\left(\hat{g}^{\prime 2}(0)+\hat{f}^{\prime 2}(0)\right)+2 \hat{h}^{\prime 2}(0)\right],} \\
\frac{C_{\hat{f}_{\theta 1}}}{\sqrt{\operatorname{Re}}} & {\left[1+(\mathrm{We}) \hat{g}^{\prime}(0)\right.} \\
& \left.\sqrt{4 \hat{f}^{2}(0)+A^{2}\left(\hat{g}^{\prime 2}(0)+\hat{f}^{\prime 2}(0)\right)+2 \hat{h}^{\prime 2}(0)}\right] .
\end{aligned}
$$

For the upper disk (radial, tangential), shear stresses and Nusselt number are given below:

$$
\begin{aligned}
C_{\hat{f}_{r 2}} & =\frac{\left.\tau_{r z}\right|_{z=h}}{\mu_{0} \Omega_{1}}, \quad C_{\breve{f}_{\hat{\theta} 2}}=\frac{\left.\tau_{\theta z}\right|_{z=h}}{\mu_{0} \Omega_{1}}, \\
\mathrm{Nu}_{r 2} & =\frac{\left.r q_{w}\right|_{z=} h}{k\left(\hat{T}_{1}-\hat{T}_{0}\right)} .
\end{aligned}
$$


Thus, the Nusselt number and skin friction for the upper disk are obtained as:

$$
\begin{aligned}
& \mathrm{Nu}_{r 2}=-\sqrt{\operatorname{Re}} \hat{\theta}^{\prime}(1), \\
& \frac{C_{\hat{f}_{r 2}}}{\sqrt{\operatorname{Re}}}=\left[1+(\mathrm{We}) \hat{f}^{\prime}(1)\right. \\
& \left.\sqrt{4 \hat{f}^{2}(1)+2 \hat{h}^{\prime 2}(1)+\operatorname{Re}\left(\hat{g}^{\prime 2}(1)+\hat{f}^{\prime 2}(1)\right)}\right], \\
& \frac{C_{\hat{f}_{\theta 2}}}{\sqrt{\operatorname{Re}}}=\left[1+(\mathrm{We}) \hat{g}^{\prime}(1)\right. \\
& \left.\sqrt{4 \hat{f}^{2}(1)+\operatorname{Re}\left(\hat{g}^{\prime 2}(1)+\hat{f}^{\prime 2}(1)\right)+2 \hat{h}^{\prime 2}(1)}\right] .
\end{aligned}
$$

For lower and upper disks, the Sherwood number can be written as follows:

$$
\mathrm{Sh}=\left.\frac{r J_{w}}{D\left(\hat{C}_{1}-\hat{C}_{0}\right)}\right|_{z=0}, \quad \mathrm{Sh}=\left.\frac{r J_{w}}{D\left(\hat{C}_{1}-\hat{C}_{0}\right)}\right|_{z=h},
$$

where $J_{w}$ is:

$$
J_{w}=-\left[D \frac{\partial \bar{C}}{\partial z}\right] \text {. }
$$

Thus, after applying transformations, the Sherwood numbers take the following form:

$$
\begin{aligned}
& \frac{\mathrm{Sh}}{\sqrt{\mathrm{Re}}}=-\hat{\varphi}^{\prime}(0), \\
& \frac{\mathrm{Sh}}{\sqrt{\mathrm{Re}}}=-\hat{\varphi}^{\prime}(1) .
\end{aligned}
$$

\section{Solution technique}

Auxiliary linear operators and initial approximations are:

$$
\begin{aligned}
& \mathbf{L}_{\hat{h}}=\hat{h}^{\prime}, \quad \mathbf{L}_{\hat{f}}=\hat{f}^{\prime \prime}, \quad \mathbf{L}_{\hat{g}}=\hat{g}^{\prime \prime}, \\
& \mathbf{L}_{\breve{\theta}}=\hat{\theta}^{\prime \prime}, \quad \mathbf{L}_{\hat{\varphi}}=\hat{\varphi}^{\prime \prime}, \\
& \hat{h}_{0}=0, \quad \hat{f}_{0}=A_{1}-A_{1} \exp (-\xi)+A_{2}(\xi), \\
& \hat{g}_{0}=1+\xi(\Omega-1), \quad \hat{\theta}_{0}=(1-\xi)\left(1-S_{1}\right), \\
& \hat{\varphi}_{0}=(1-\xi)\left(1-S_{2}\right), \\
& \text { with }, \\
& \mathbf{L}_{\hat{h}}\left[Z_{1}\right]=0, \\
& \mathbf{L}_{\breve{f}}\left[Z_{2}+Z_{3} \xi\right]=0, \\
& \mathbf{L}_{\hat{g}}\left[Z_{4}+Z_{5} \xi\right]=0 \\
& \mathbf{L}_{\hat{\theta}}\left[Z_{6}+Z_{7} \xi\right]=0 \\
& \mathbf{L}_{\hat{\varphi}}\left[Z_{8}+Z_{9} \xi\right]=0
\end{aligned}
$$

where the constants are $Z_{i}(i=1-5)$.

\section{Convergence}

Auxiliary variables $\left(\hbar_{\hat{g}}, \hbar_{\hat{\varphi}}, \hbar_{\hat{\theta}}, \hbar_{\hat{h}}, \hbar_{\hat{f}}\right)$, play a prominent role in the convergence analysis. Figure 2 shows $\hbar-$ curves for the $m$ th-order approximation. The solutions are found to be convergent for the regions $-1.7 \leq$ $\hbar_{\hat{h}} \leq-0.3,-1.2 \leq \hbar_{\hat{f}} \leq-0.3,-1.0 \leq \hbar_{\hat{g}} \leq-0.4$, $-0.9 \leq \hbar_{\hat{\theta}} \leq-0.6$, and $-1.0 \leq \hbar_{\hat{\varphi}} \leq-0.7$. Table 1 comprises numerical values of velocity, temperature, and concentration. Table 1 shows the numerical values of velocity concentration and temperature distribution. Clearly, a meaningful solution of $\hat{h}^{\prime}(0), \hat{f}^{\prime}(0), \hat{g}^{\prime}(0)$, $\hat{\theta}^{\prime}(0)$, and $\hat{\varphi}^{\prime}(0)$ is derived from the 20th-order approximations. Table 2 shows good agreement between the obtained results and those in the previous literature.

\section{Entropy}

By considering the roles of thermal irreversibility, Joule heating irreversibility, viscous dissipation irreversibility, and mass transfer irreversibility, the formulation of entropy generation is presented. Its dimensional form is represented by Eq. (35) as shown in Box II, where $\hat{C}_{m}$ and $\hat{T}_{m}$ are mean concentration and temperature, respectively, and $(\nabla \hat{T}),(\nabla \hat{C})$ and $(\Phi)$ are defined below:

$$
\begin{aligned}
& \nabla \hat{T}=\left(\frac{\partial \hat{T}}{\partial r}\right) \hat{e}_{r}+\left(\frac{1}{r} \frac{\partial \hat{T}}{\partial \theta}\right) \hat{e}_{\theta}+\left(\frac{\partial \hat{T}}{\partial z}\right) \hat{e}_{z} \\
& \nabla \hat{C}=\left(\frac{\partial \hat{C}}{\partial r}\right) \hat{e}_{r}+\left(\frac{1}{r} \frac{\partial \hat{C}}{\partial \theta}\right) \hat{e}_{\theta}+\left(\frac{\partial \hat{C}}{\partial z}\right) \hat{e}_{z} \\
& \Phi=\mu_{0}\left[\left(\frac{\partial u}{\partial y}\right)^{2}+\Gamma\left(\frac{\partial u}{\partial y}\right)^{3}\right]
\end{aligned}
$$

After applying transformation, one obtains the

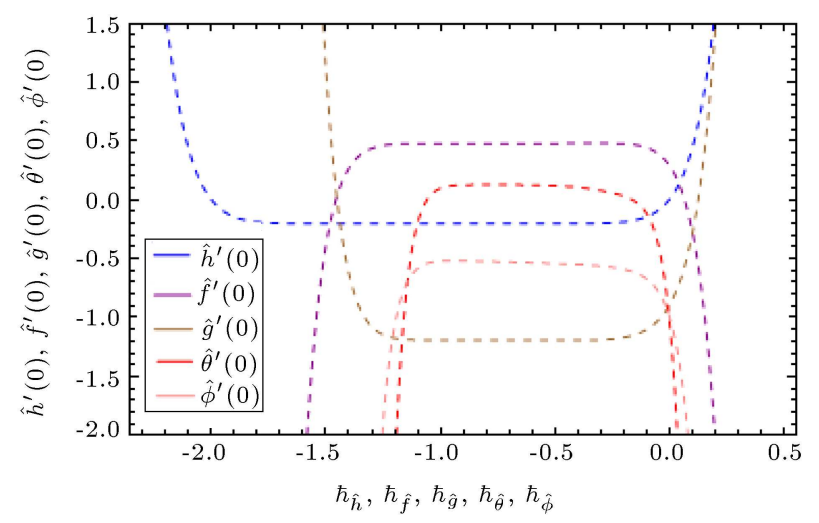

Figure 2. $\hbar$-curves for axial, radial, tangential velocity, temperature, and concentration profiles. 
Table 1. Series solutions convergence for $\Omega=0.2, n_{1}=1, A_{1}=0.1, A_{2}=0.4, n=0.1, E_{1}=0.5, \sigma=0.5, \delta=0.5$, $S_{1}=S_{2}=0.02, D_{f}=0.5, E c=0.1, M=0.7, \mathrm{Sc}=1, \mathrm{Re}=0.7, \mathrm{We}=0.2, \mathrm{Pr}=0.6, \mathrm{Sr}=0.3$, $\hbar_{\hat{h}}=\hbar_{\hat{f}}=\hbar_{\hat{g}}=\hbar_{\hat{\theta}}=\hbar_{\hat{\varphi}}=-0.8$.

\begin{tabular}{cccccc}
\hline $\begin{array}{c}\text { Order of } \\
\text { approximation }\end{array}$ & $-\hat{\boldsymbol{h}}(\mathbf{0})$ & $\hat{\boldsymbol{f}}^{\prime}(\mathbf{0})$ & $-\hat{\boldsymbol{g}}^{\prime}(\mathbf{0})$ & $\hat{\boldsymbol{\theta}}^{\prime} \mathbf{( 0 )}$ & $-\boldsymbol{\varphi}^{\prime}(\mathbf{0})$ \\
\hline 1 & 0.20000 & 0.5272 & 1.0380 & -0.11008 & 0.6884 \\
5 & 0.20000 & 0.4809 & 1.192 & -0.09493 & 0.6863 \\
11 & 0.20000 & 0.4812 & 1.192 & 0.09682 & 0.5462 \\
16 & 0.20000 & 0.4812 & 1.192 & 0.2655 & 0.4127 \\
20 & 0.20000 & 0.4812 & 1.192 & 0.2655 & 0.3554 \\
25 & 0.20000 & 0.4812 & 1.192 & 0.2655 & 0.3554 \\
30 & 0.20000 & 0.4812 & 1.192 & 0.2655 & 0.3554 \\
40 & 0.20000 & 0.4812 & 1.192 & 0.2655 & 0.3554 \\
\hline
\end{tabular}

$$
S_{G}^{\prime \prime}=\underbrace{\frac{\tilde{\mu}}{\hat{T}_{m}} \Phi}_{\text {Viscous dissipation irreversibility }}+\underbrace{\frac{k}{\hat{T}_{m}^{2}}(\nabla \hat{T})^{2}}_{\text {Thermal irreversibility Joule dissipation irreversibility }}+\underbrace{\frac{\sigma}{\hat{T}_{m}} B_{0}^{2}\left(\hat{u}^{2}+\hat{v}^{2}\right)}_{\text {Mass transfer irreversibility }}+\underbrace{\frac{R_{g} D}{\hat{C}_{m}}[\nabla \hat{C}]^{2}+\frac{D R_{g}}{\hat{T}_{m}}[\nabla \hat{C} \cdot \nabla \hat{T}]}_{\text {C. }} .
$$

Box II

Table 2. Comparison table for the validation of the problem with Ref. [5] by varying We.

\begin{tabular}{ccccc}
\hline We & $\begin{array}{c}\boldsymbol{C}_{\boldsymbol{f o}} \\
{[\mathbf{5}]}\end{array}$ & $\begin{array}{c}-\boldsymbol{C}_{\boldsymbol{g o}} \\
{[\mathbf{5}]}\end{array}$ & $\begin{array}{c}\boldsymbol{C}_{\boldsymbol{f o}} \\
{[\text { Present }]}\end{array}$ & $\begin{array}{c}-\boldsymbol{C}_{\boldsymbol{g o}} \\
{[\text { Present }]}\end{array}$ \\
\hline 0.1 & 0.550420 & 1.12934 & 0.550420 & 1.12934 \\
0.2 & 0.590397 & 1.20342 & 0.590397 & 1.20342 \\
0.3 & 0.630044 & 1.27751 & 0.630044 & 1.27751 \\
\hline
\end{tabular}

following:

$$
\begin{aligned}
N_{G}= & \alpha_{1}\left[\operatorname{Re} \hat{\theta}^{\prime 2}+\left(S_{1}+\theta\right)^{2}\right] \\
& +M(\operatorname{Re})(B r)\left(\hat{f}^{2}+\hat{g}^{2}\right)+(B r)(1+\mathrm{We} \dot{\gamma}) \\
& {\left[4 \hat{f}^{2}+\operatorname{Re} \hat{f}^{\prime 2}+\operatorname{Re} \hat{g}^{\prime 2}+2 \hat{h}^{\prime 2}\right] } \\
& +L\left[\frac{\alpha_{2}}{\alpha_{1}}\right]\left[\operatorname{Re} \hat{\varphi}^{\prime 2}+\left(\hat{\varphi}+S_{1}\right)^{2}\right] \\
& +L\left[\left(\hat{\theta}+S_{1}\right)\left(S_{2}+\hat{\varphi}\right)+\operatorname{Re} \hat{\varphi}^{\prime} \hat{\theta}^{\prime}\right] .
\end{aligned}
$$

Dimensionless parameters are given as follows:

$$
\alpha_{1}=\frac{\hat{T}_{1}-\hat{T}_{0}}{\hat{T}_{m}}, \quad L=\frac{R_{g} D\left(\hat{C}_{1}-\hat{C}_{0}\right)}{k},
$$

$$
\begin{aligned}
B r & =\frac{\tilde{\mu}_{0} r^{2} \Omega_{1}^{2}}{k\left(\hat{T}_{1}-\hat{T}_{0}\right)}, \quad N_{G}=\frac{r^{2} \hat{T}_{m} S_{G}^{\prime \prime}}{k\left(\hat{T}_{1}-\hat{T}_{0}\right)}, \\
\alpha_{2} & =\frac{\hat{C}_{1}-\hat{C}_{0}}{\hat{C}_{m}},
\end{aligned}
$$

where $\mathrm{Br}$ and $N_{G}$ are Brinkman number and local entropy generation, respectively, $\alpha_{1}$ and $\alpha_{2}$ are temperature and concentration ratio parameters, and $L$ is the diffusion parameter.

Herein, the dimensionless form of Bejan number (Be) is given by Eqs. (41) and (42) as shown in Box III.

\section{Discussion}

This section discusses a graphical interpretation of various physical parameters for velocity, temperature, entropy generation, Bejan number, skin friction, Nusselt number, and Sherwood number.

\subsection{Velocity profile}

Figures 3-8 are designed to analyze the behavior of Hartmann number $(M)$ and Weissenberg number (We) on $\hat{h}(\xi), \hat{f}(\xi)$, and $\hat{g}(\xi)$ in axial, radial, and transverse directions. These quantities are discussed for both upper and lower disks. Figures $3-5$ show the analysis of the behavior of $M$ for axial $\hat{h}(\xi)$, radial $\hat{f}(\xi)$, and tangential $\hat{g}(\xi)$ velocities at both disks. It is found that the magnitude of velocities $\hat{h}(\xi), \hat{f}(\xi)$, and $\hat{g}(\xi)$ is reduced as the Hartman number $(M)$ 
$\mathrm{Be}=\frac{\text { Entropy generation due to heat and mass transfer }}{\text { Total entropy generation }}$,

$$
\begin{gathered}
\mathrm{Be}=\frac{\alpha_{1}\left[\operatorname{Re} \hat{\theta}^{\prime 2}+\left(S_{1}+\theta\right)^{2}\right]+L\left[\frac{\alpha_{2}}{\alpha_{1}}\right]\left[\operatorname{Re} \hat{\varphi}^{\prime 2}+\left(S_{1}+\hat{\varphi}\right)^{2}\right]+L\left[\left(S_{1}+\hat{\theta}\right)\left(S_{2}+\hat{\varphi}\right)+\operatorname{Re} \hat{\varphi}^{\prime} \hat{\theta}^{\prime}\right]}{L\left[\left(S_{1}+\hat{\theta}\right)\left(S_{2}+\hat{\varphi}\right)+\operatorname{Re} \hat{\varphi}^{\prime} \hat{\theta}^{\prime}\right]+M(\operatorname{Re})(B r)\left(\hat{f}^{2}+\hat{g}^{2}\right)+\alpha_{1}\left[\left(S_{1}+\theta\right)^{2}+\operatorname{Re} \hat{\theta}^{\prime 2}\right]} . \\
+L\left[\frac{\alpha_{2}}{\alpha_{1}}\right]\left[\left(S_{1}+\hat{\varphi}\right)^{2}+\operatorname{Re} \hat{\varphi}^{\prime 2}\right]+(1+\operatorname{We} \dot{\gamma})(B r)\left[4 \hat{f}^{2}+\operatorname{Re}\left(\hat{f}^{\prime 2}+\hat{g}^{\prime 2}\right)+2 \hat{h}^{\prime 2}\right]
\end{gathered}
$$

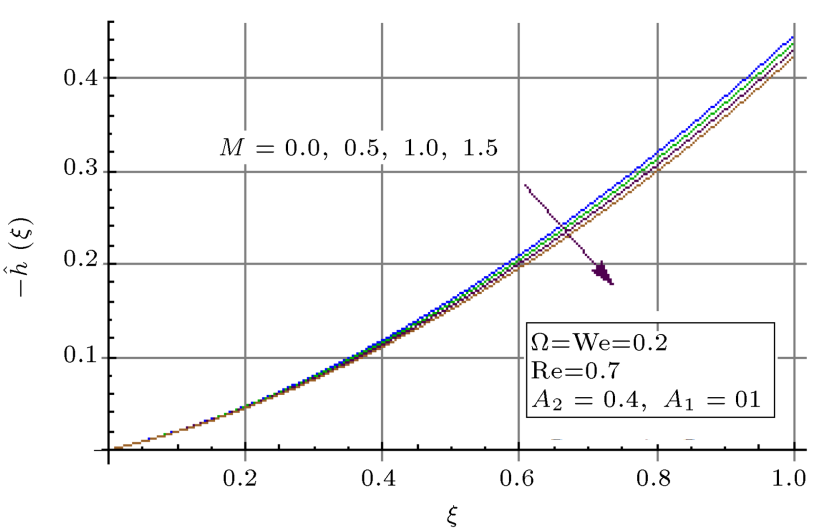

Figure 3. Axial velocity $\hat{h}(\xi)$ via $M$.

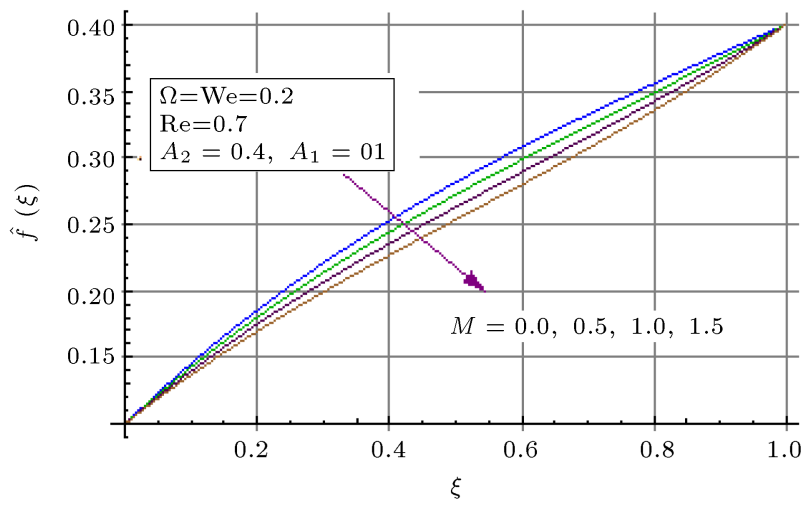

Figure 4. Radial velocity $\hat{f}(\xi)$ via $M$.

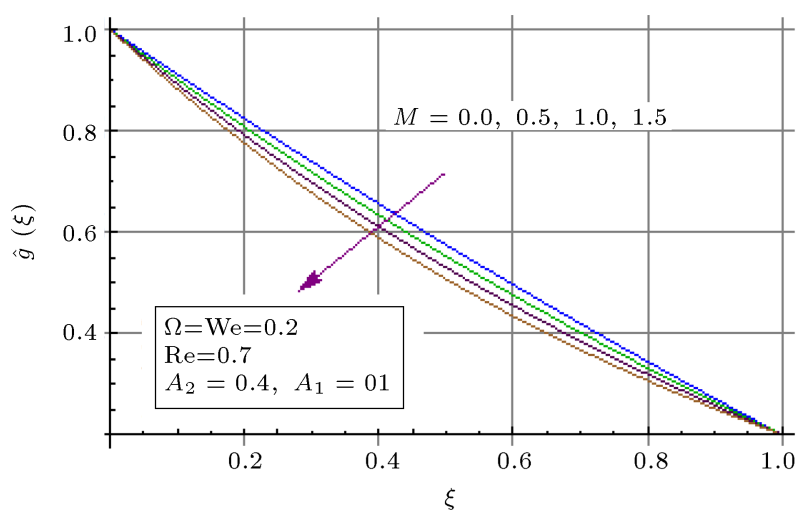

Figure 5. Tangential velocity $\hat{g}(\xi)$ via $M$.

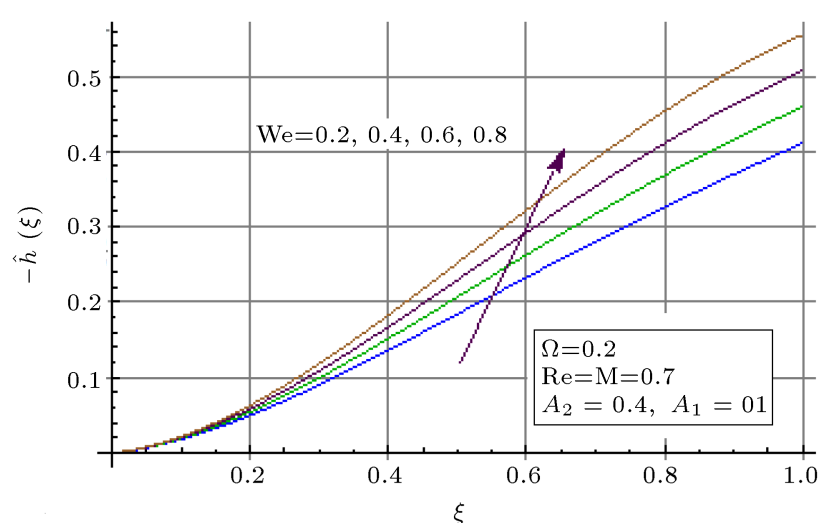

Figure 6. Axial velocity $\hat{h}(\xi)$ via We.

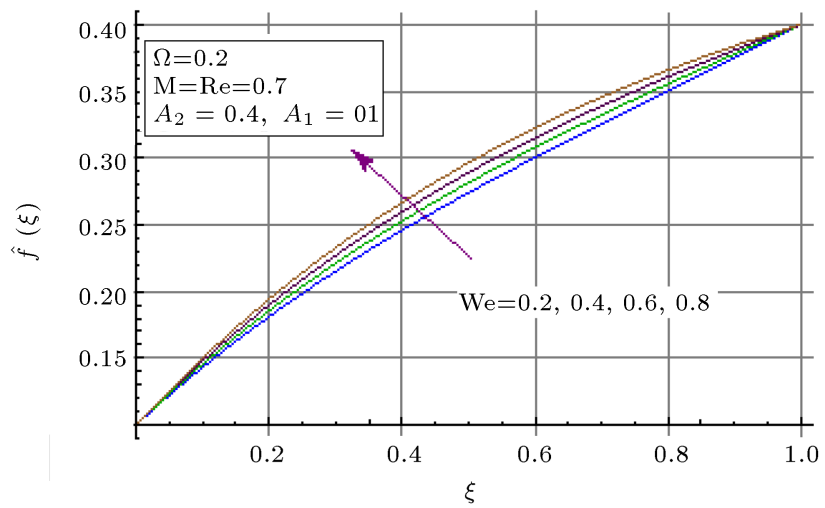

Figure 7. Radial velocity $\hat{f}(\xi)$ for We.

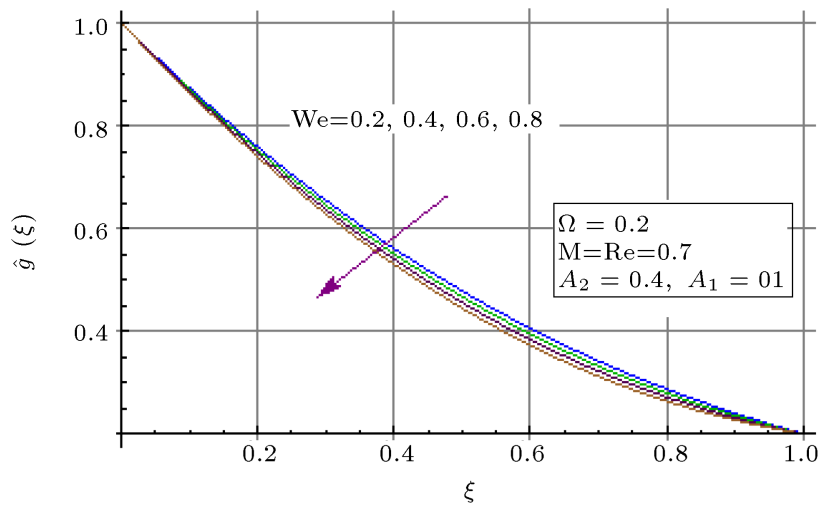

Figure 8. Tangential velocity $\hat{g}(\xi)$ via We. 
increases. A resistive force is produced when the transverse magnetic field is in effect. A force acting as Lorentz force generates resistance and reduces velocity. The effect of Weissenberg number (We) on $\hat{h}(\xi), \hat{f}(\xi)$, and $\hat{g}(\xi)$ is shown in Figures $6-8$. The axial and radial velocities, $\hat{h}(\xi)$, and $\hat{f}(\xi)$, rise when Weissenberg number (We) increases and tangential velocity $\hat{g}(\xi)$, decreases. At a higher We number, the rotational velocity $\left(\Omega_{1}\right)$ improves and, thus, $\hat{h}(\xi)$, and $\hat{f}(\xi)$ increase (see Figures 6 and 7 ).

\subsection{Temperature}

Figures 9-12 illustrate the trend of temperature $(\hat{\theta}(\xi))$ against Dufour number $\left(D_{1}\right)$, thermal stratification parameter $\left(S_{1}\right)$, Weissenberg number (We), and Prandtl number $(\mathrm{Pr})$. Figure 9 shows the impact of $D_{1}$ on $\hat{\theta}(\xi)$ at lower and upper disks. Fluid temperature via $D_{1}$ improves. Energy flux is enhanced due to increase in the concentration gradient for varying $D_{1}$ and it leads to the enhancement of the fluid temperature. Temperature close to the lower disk is higher than that to the upper disk because of the higher temperature of the lower disk than the upper disk, i.e., $\hat{T}_{1} \succ \hat{T}_{2}$. Figure 10 shows the impact of thermal stratification parameter $S_{1}$ on $\hat{\theta}(\xi)$. To estimate the large number of $S_{1}$, the temperature decreases due to a potential drop

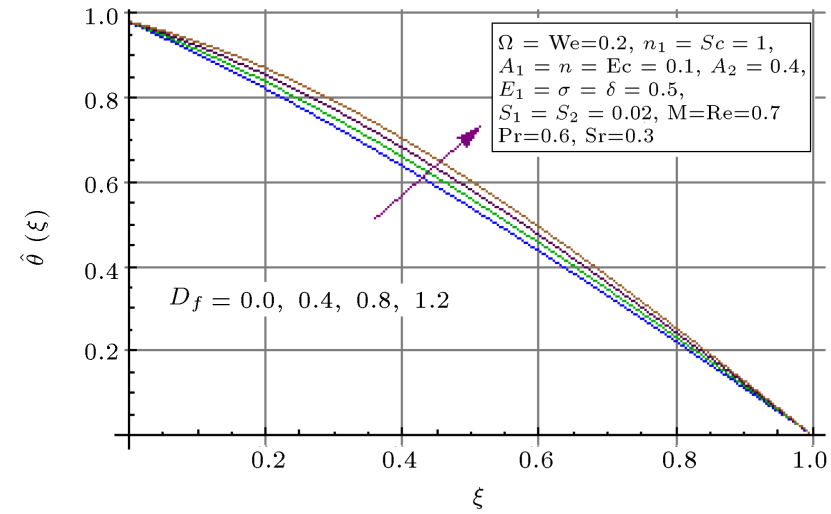

Figure 9. Temperature profile via $D_{f}$.

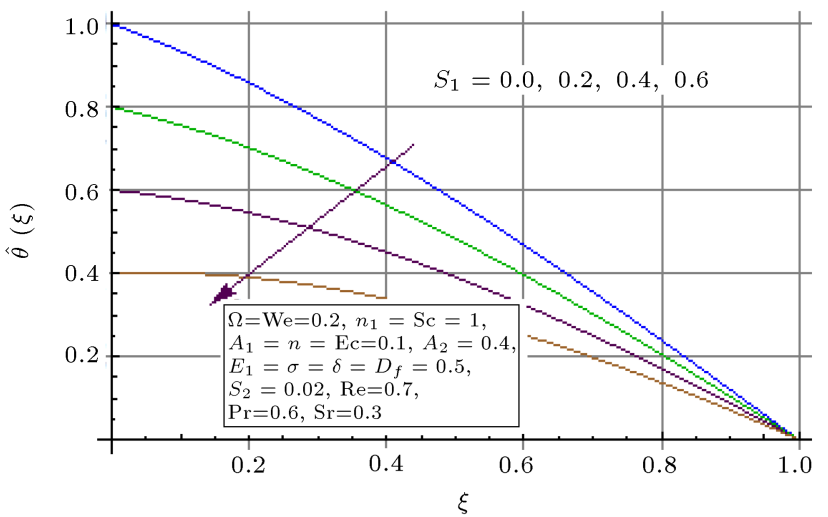

Figure 10. Temperature profile via $S_{1}$. between the ambient fluid temperature and the surface condition. Figure 11 shows the effect of Weissenberg number We, on $\hat{\theta}(\xi)$. Temperature is enhanced in the case of estimating higher $S_{1}$. The behavior of Prandtl number, Pr, with respect to $\hat{\theta}(\xi)$ is shown in Figure 12. For larger Pr, thermal diffusivity has a smaller value, which causes a reduction in temperature $\hat{\theta}(\xi)$.

\subsection{Concentration}

Figures $13-18$ show the trend of concentration $(\hat{\varphi}(\xi))$ for variations of Schmidt number (Sc), Soret number $(\mathrm{Sr})$, dimensionless activation energy parameter $\left(E_{1}\right)$,

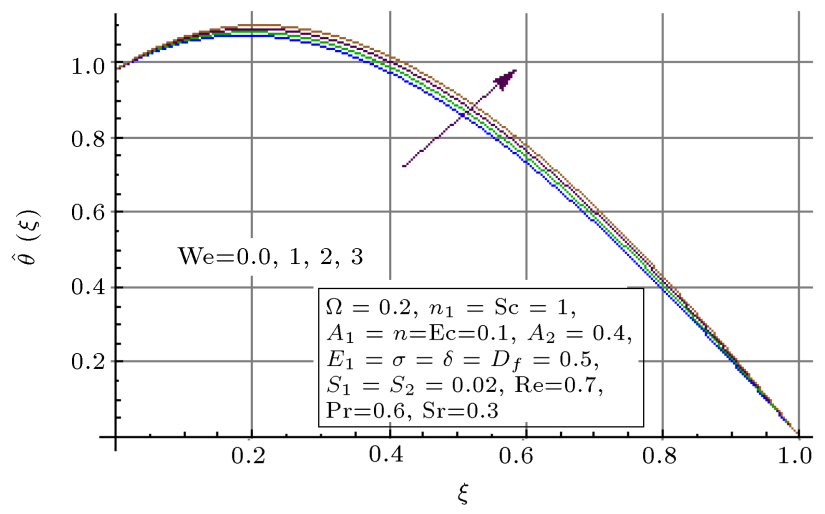

Figure 11. Temperature profile via We.

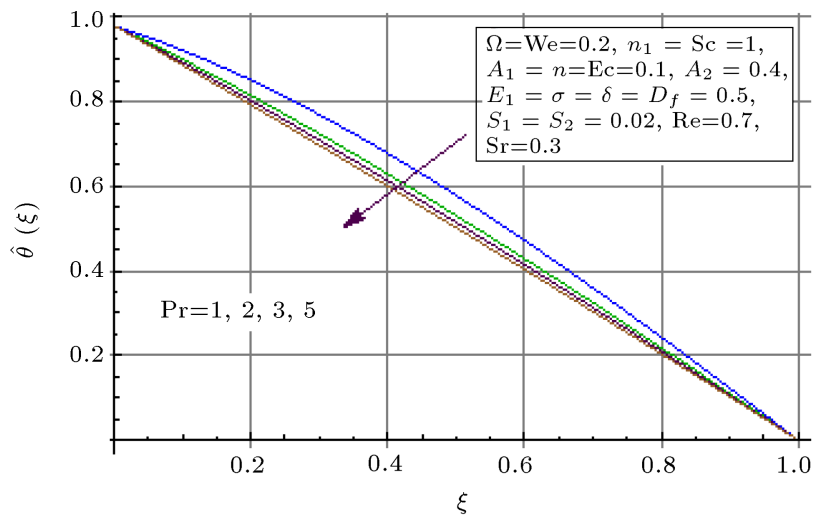

Figure 12. Temperature profile via Pr.

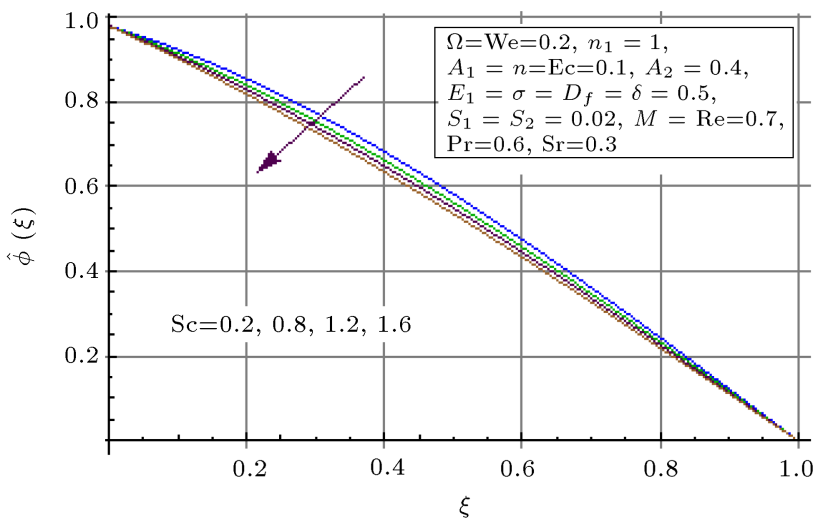

Figure 13. Temperature profile via Sc. 


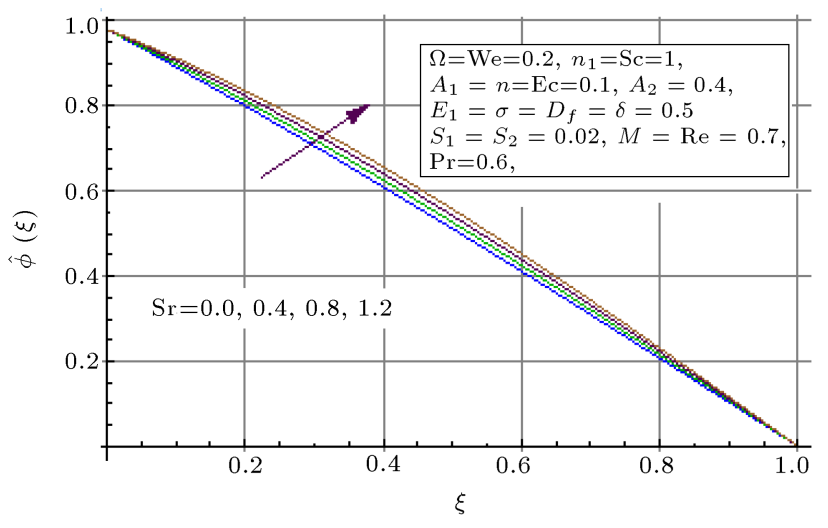

Figure 14. Concentration profile via Sr.

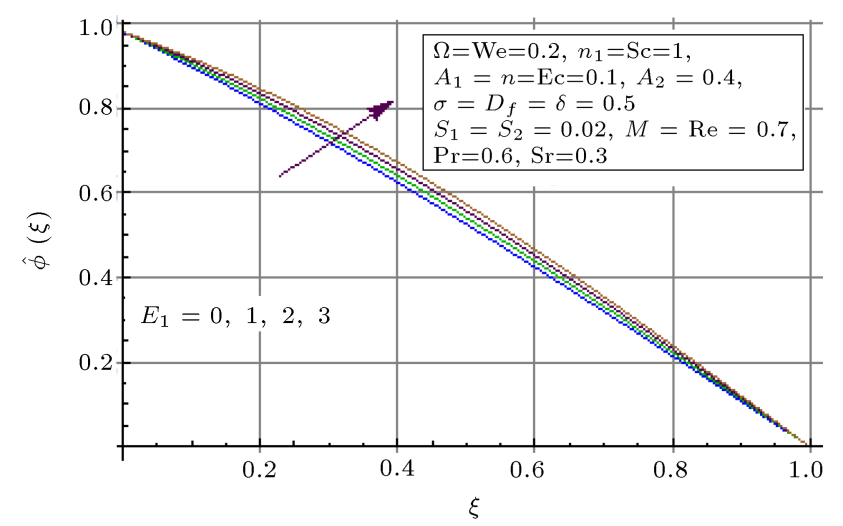

Figure 15. Temperature profile via $E_{1}$.

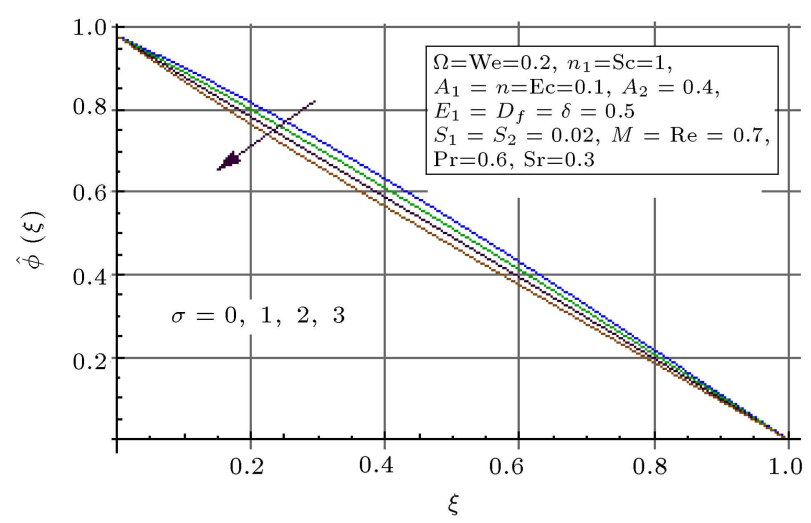

Figure 16. Temperature profile via $\sigma$.

dimensionless reaction rate $(\sigma)$, temperature difference parameter $(\delta)$, and solutal stratification parameter $\left(S_{2}\right)$. At the growing values of $\mathrm{Sc}$, the concentration $(\hat{\varphi}(\xi))$ decreases because mass diffusivity is reduced for larger Sc (see Figure 13). The effect of Soret number $(\mathrm{Sr})$ on $\hat{\varphi}(\xi)$ is shown in Figure 14. Accordingly, the concentration $\hat{\varphi}(\xi)$ increases for larger Sr. Temperature gradient is enhanced for larger Sr, which tends towards greater convective flow. Hence, the concentration distribution $\hat{\varphi}(\xi)$ is enhanced. Figure 15 shows that the concentration $\hat{\varphi}(\xi)$ increases for the larger dimensionless activation energy parameter $\left(E_{1}\right)$.

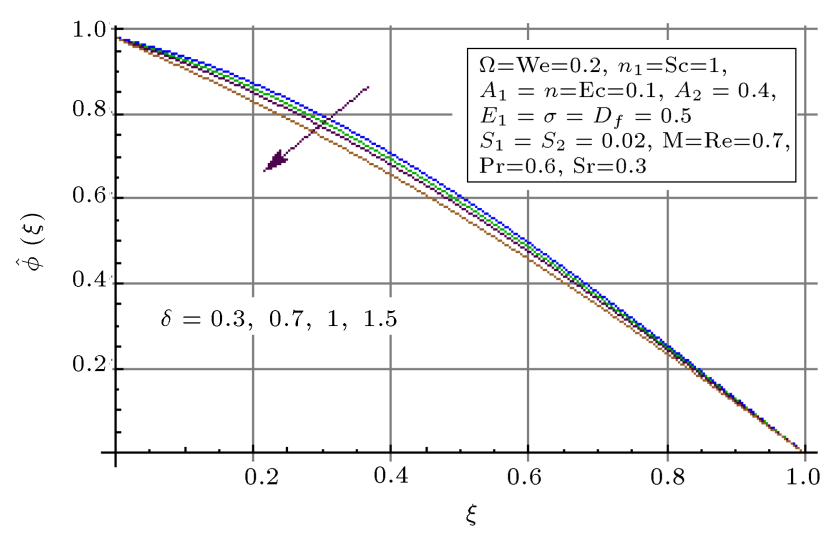

Figure 17. Concentration profile via $\delta$.

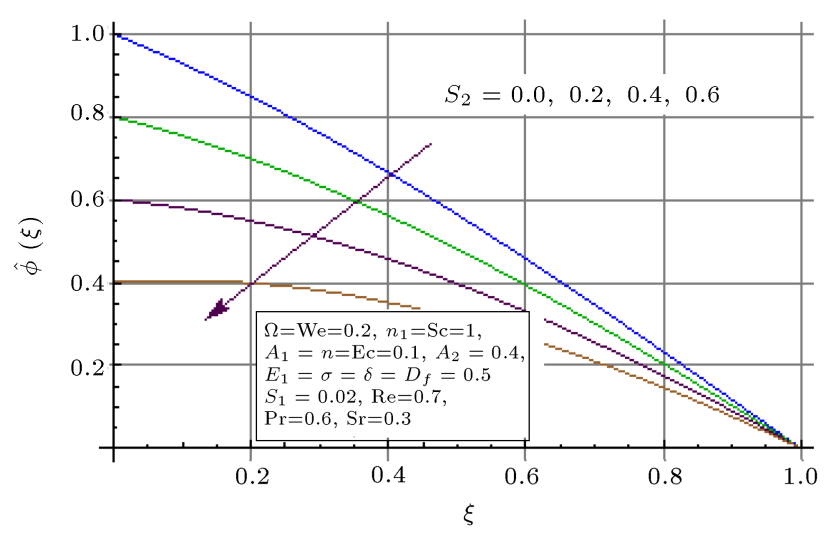

Figure 18. Concentration profile via $S_{2}$.

Generative chemical reaction is promoted due to a decrease in Arrhenius function $\left(\frac{\hat{T}}{\hat{T}_{\infty}}\right) \exp \left(\frac{-E_{\alpha}}{\kappa \hat{T}}\right)$. When $E_{\alpha}$ increases, concentration profile $\hat{\varphi}(\xi)$ is enhanced. Figure 16 analyzes the effect of chemical reaction parameter $\sigma$ on $\hat{\varphi}(\xi)$. For larger $\sigma$, a reduction in the concentration $\hat{\varphi}(\xi)$ is noticed. Due to concentration $\hat{\varphi}(\xi)$ gradient, this behavior shows weak buoyancy effect; thus, a reduction in $\hat{\varphi}(\xi)$ occurs. Figure 17 shows that the concentration $\hat{\varphi}(\xi)$ is reduced for the larger temperature difference parameter $\delta$. According to Figure 18, the concentration $\hat{\varphi}(\xi)$ is the decreasing function of the larger solutal stratification parameter $S_{2}$.

\subsection{Entropy generation minimization}

This section emphasizes the graphical interpretation of various physical parameters of entropy generation $\left(N_{g}\right)$ and Bejan number $(\mathrm{Be})$. Br effects on $N_{g}$ and Be are shown in Figures 19 and 20. The opposite trend for $\mathrm{Be}$ is observed due to a increment in the disorderness of system for larger Be. For a larger estimation of $\mathrm{Br}$ due to its dissipation, a lower rate of conduction is produced and, thus, entropy generation $\left(N_{g}\right)$ is enhanced. The impact of diffusion $L$ on $N_{g}$ and $\mathrm{Be}$ is shown in Figures 21 and 22. Both $N_{g}$ and $\mathrm{Be}$ experience an increase for larger $L$. For the 


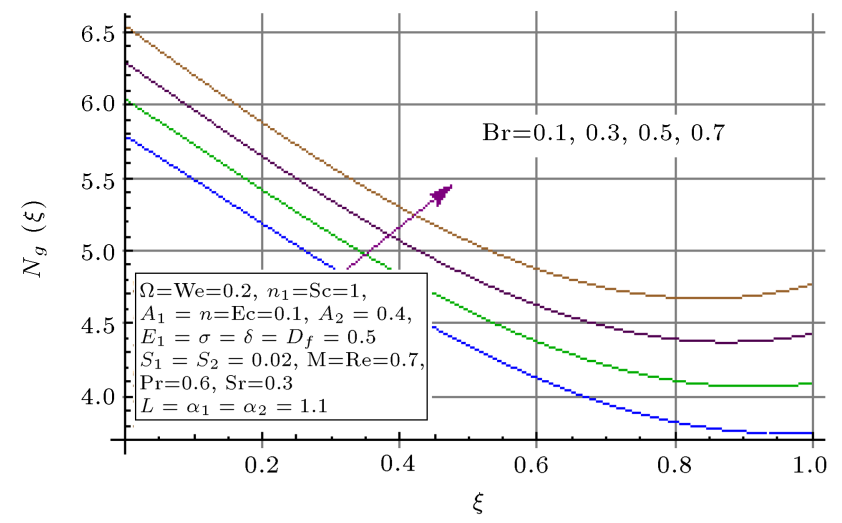

Figure 19. Entropy generation $\left(N_{g}\right)$ via $\mathrm{Br}$.

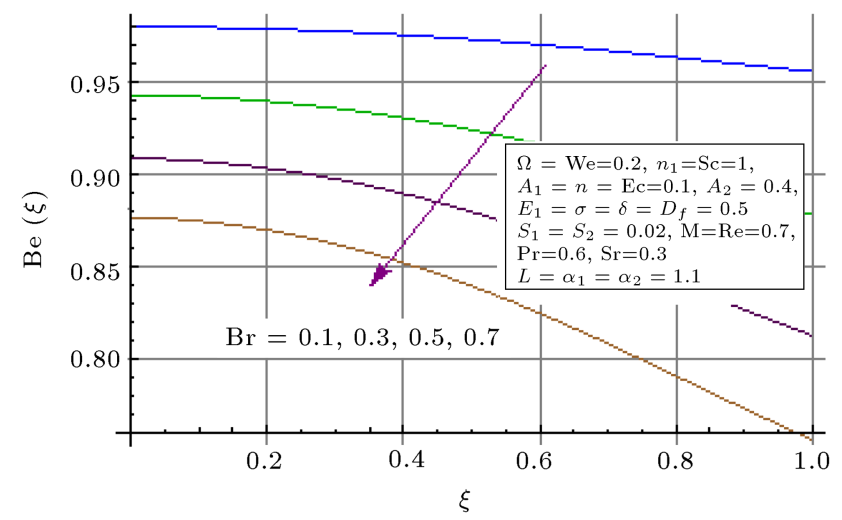

Figure 20. Bejan number $(\mathrm{Be})$ via $\mathrm{Br}$.

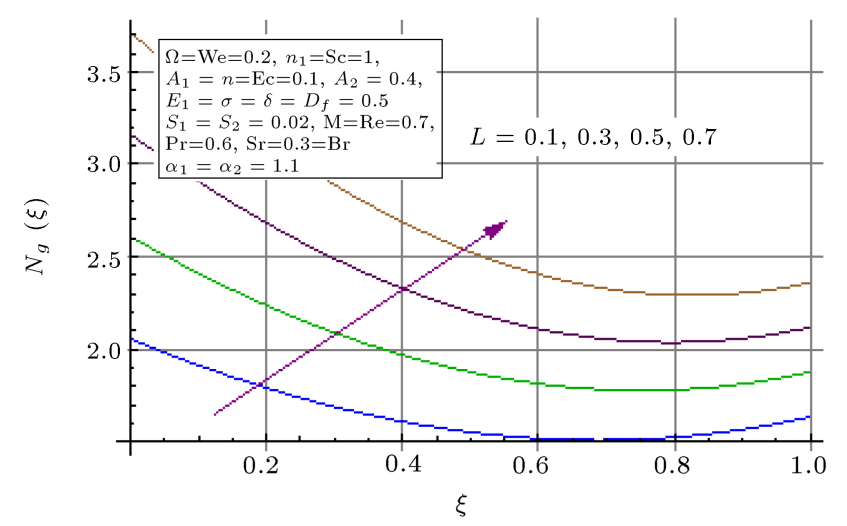

Figure 21. Entropy generation $\left(N_{g}\right)$ via $L$.

increasing $L$, the diffusion rate of nanoparticles is enhanced; therefore, Bejan number and total entropy of the system increase. The effect of We on $N_{g}$ and Be is discussed in Figures 23 and 24. For larger We, the entropy generation increases, while Bejan number decreases. Figures 25-28 show the impact of solutal and thermal stratification parameters $S_{2}$ and $S_{1}$ on $N_{g}$ and Be. Both $N_{g}$ and Be are reduced for the larger estimation of $S_{1}$ and $S_{2}$. Figures 29 and 30 clearly reveal the effect of $M$ on $N_{g}$, and the enhancement of Be in $N_{g}$ parallel to increase in the magnetic parameter

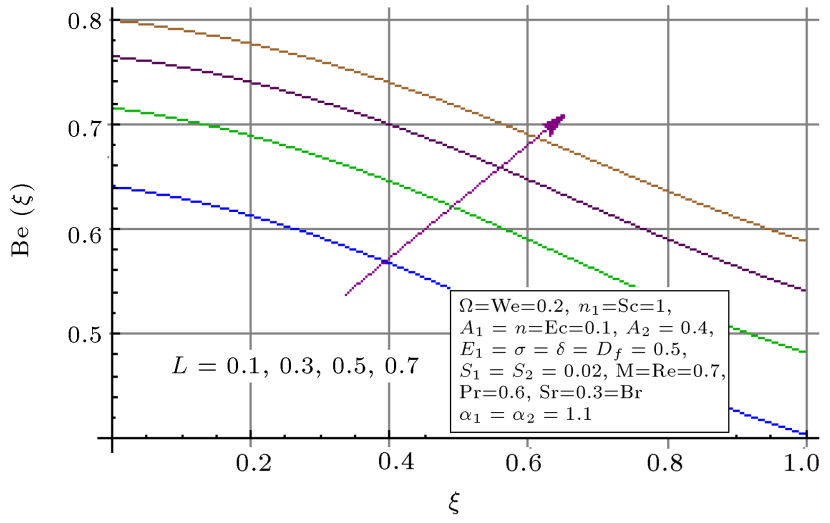

Figure 22. Bejan number $(\mathrm{Be})$ via $L$.

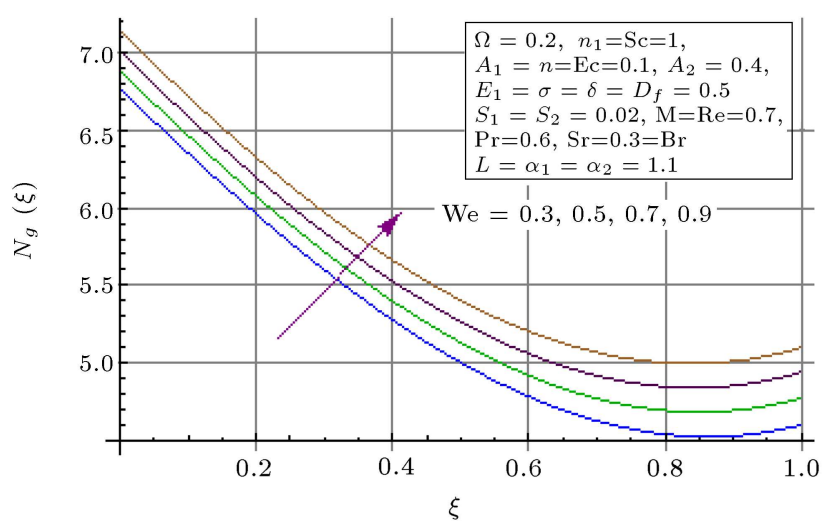

Figure 23. Entropy generation $\left(N_{g}\right)$ via We.

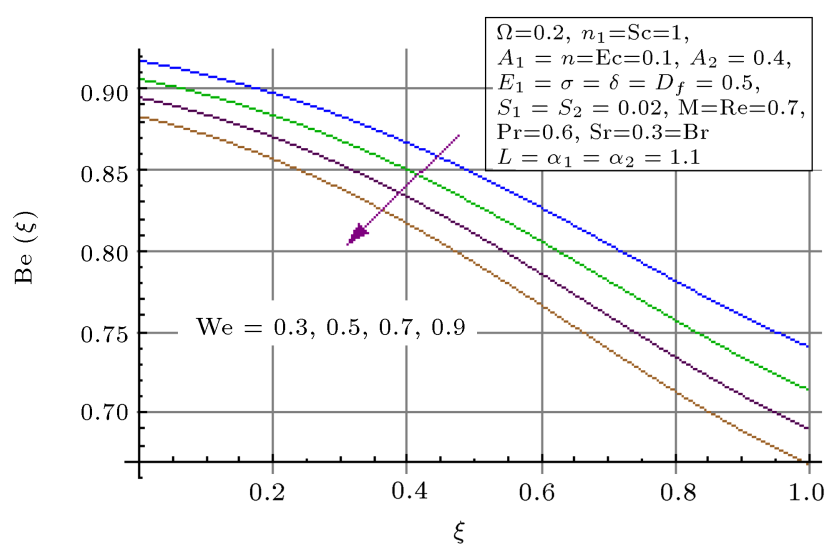

Figure 24. Bejan number $(\mathrm{Be})$ via We.

$(M)$ is witnessed. For larger $M$, the fluid resistance grows due to rise in Lorentz force and, consequently, $N_{g}$ increases. Bejan number (Be) reduces for larger $M$. Herein, the irreversibility of fluid friction prevailed over the heat and mass transfer irreversibilities.

\subsection{Skin friction and Nusselt and Sherwood numbers}

Effects of Reynold number (Re) on skin friction coefficient at lower and upper disks are analyzed in Figures 29 and 30 . There is an increase in skin friction 


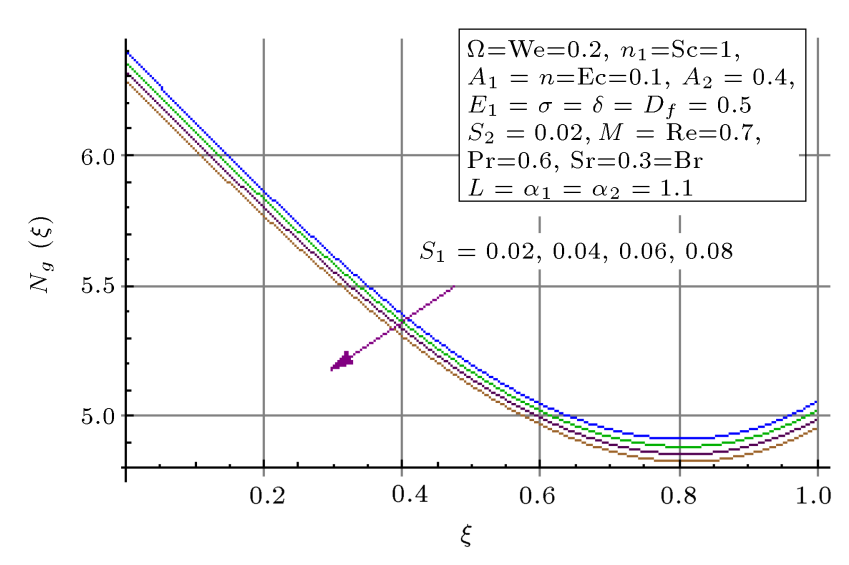

Figure 25. Entropy generation $\left(N_{g}\right)$ via $S_{1}$.

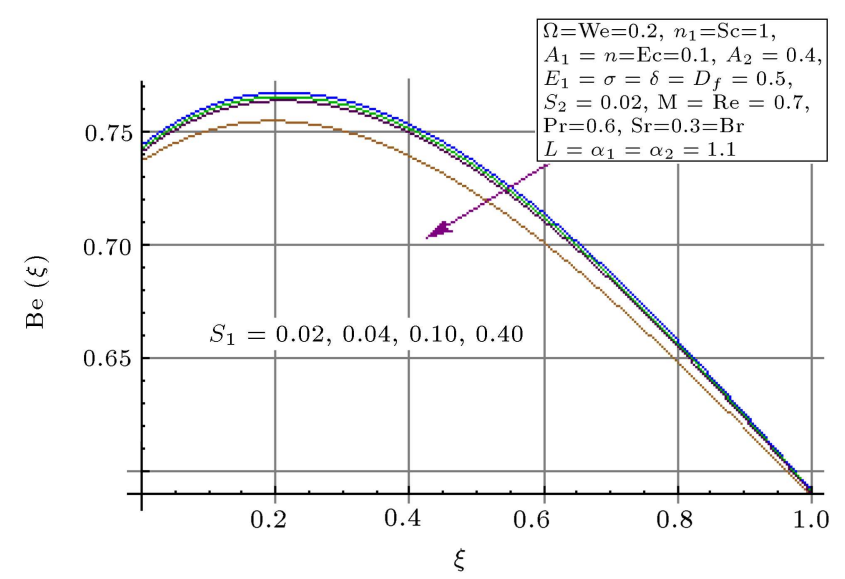

Figure 26. Bejan number $(\mathrm{Be})$ via $S_{1}$.

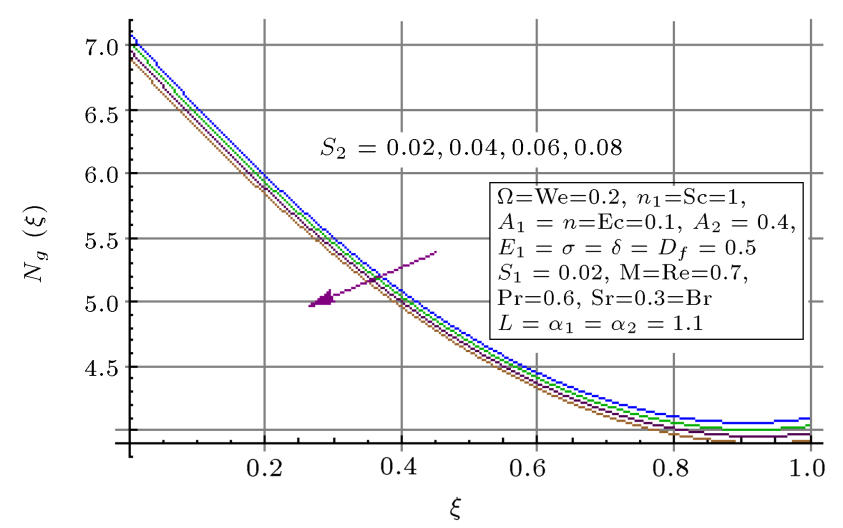

Figure 27. Entropy generation $\left(N_{g}\right)$ via $S_{2}$.

at both disks for the larger Re. From Figures 31 and 32, for the increasing Eckert number (Ec) the heat transfer rate near the surface of lower disk decreases, while reverse behavior is observed for the upper disk. Figures 33 and 34 are drawn to investigate the effect of stratification parameter $\left(S_{2}\right)$ on the Sherwood number. For varying $S_{2}$, the Sherwood number near the surface of the lower disk is reduced. However, in proximity to the upper disk, the Sherwood number experiences a reverse behavior.

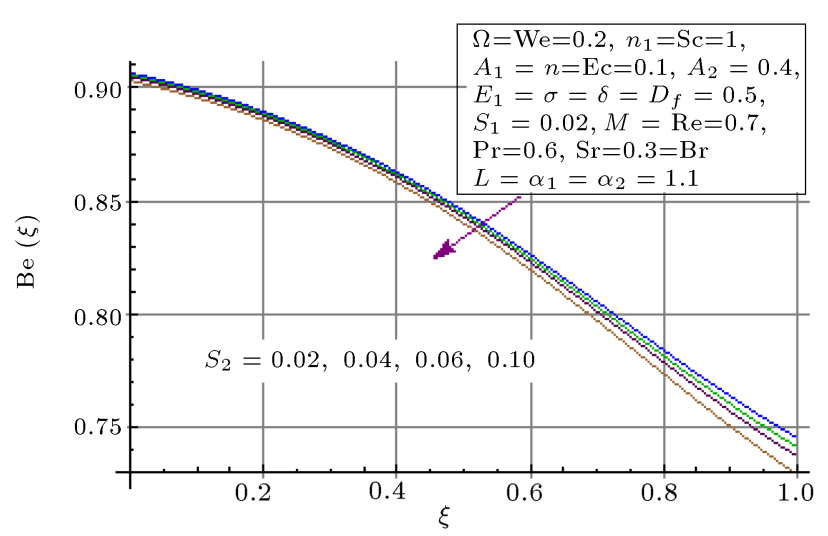

Figure 28. Bejan number (Be) via $S_{2}$.

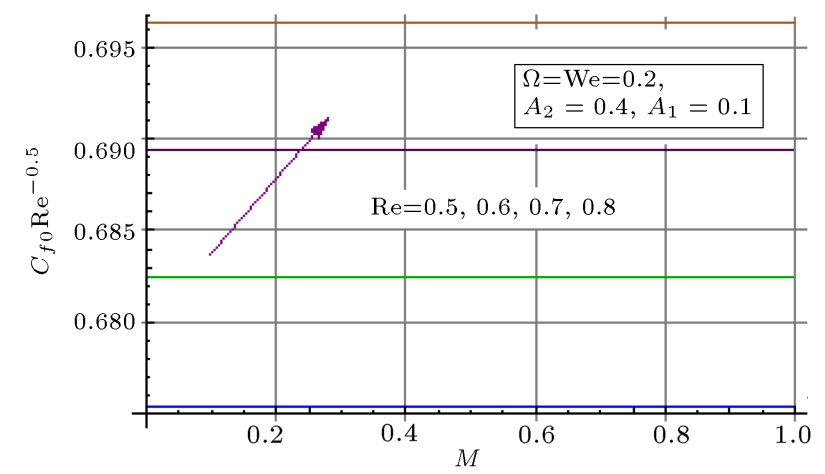

Figure 29. Skin friction via Re.

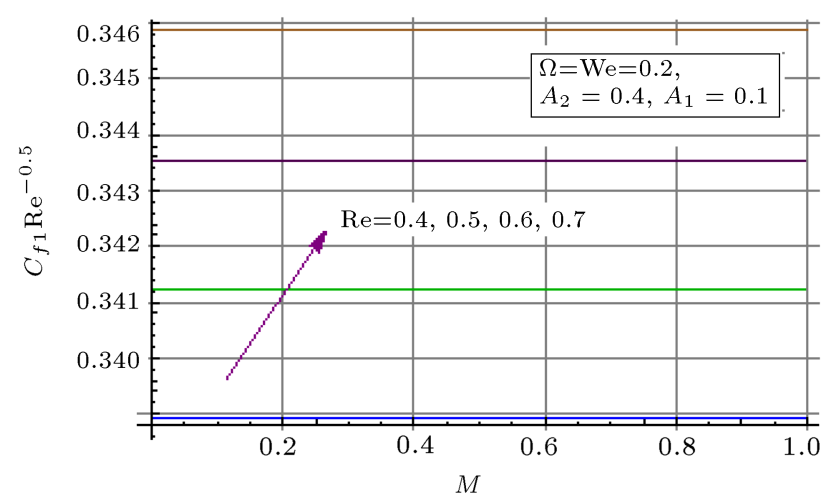

Figure 30. Skin friction via Re.

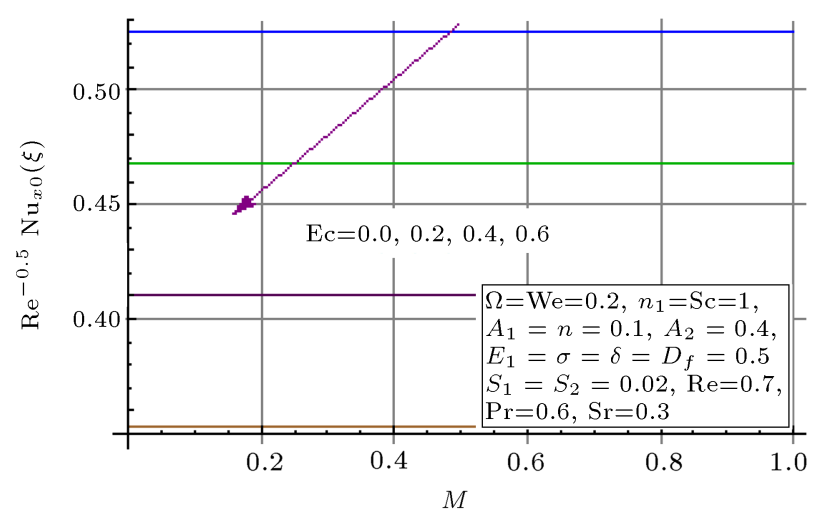

Figure 31. Nusselt number via Ec at lower disk. 


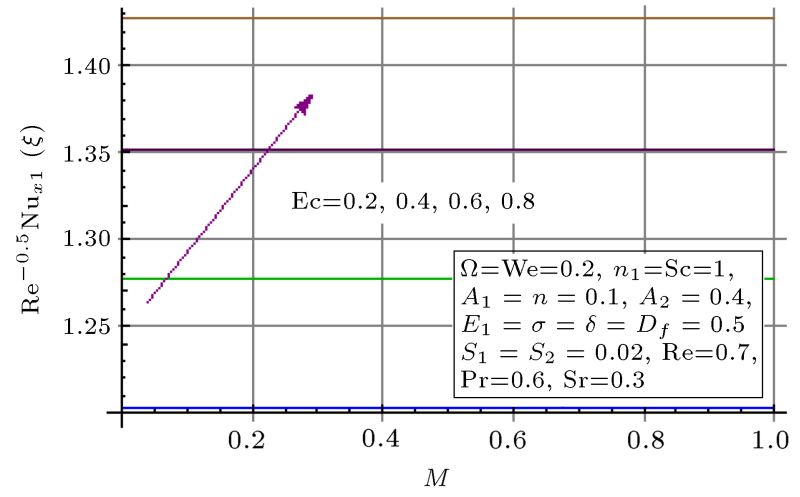

Figure 32. Nusselt number via Ec at upper disk.

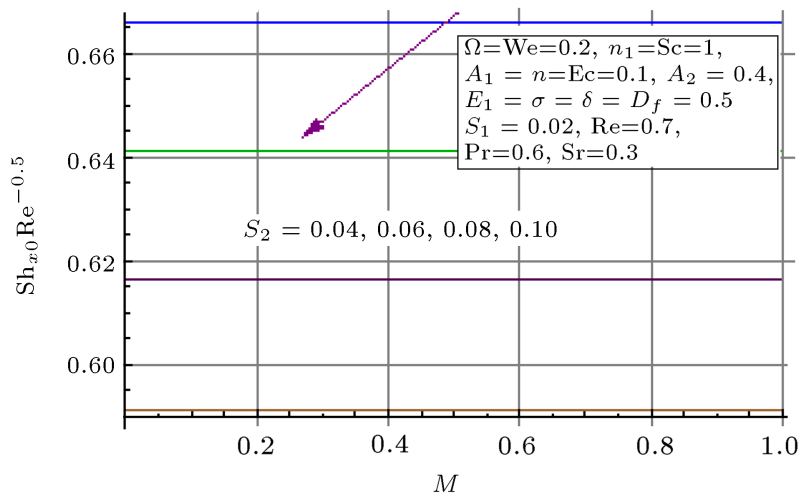

Figure 33. Sherwood number via $S_{2}$.

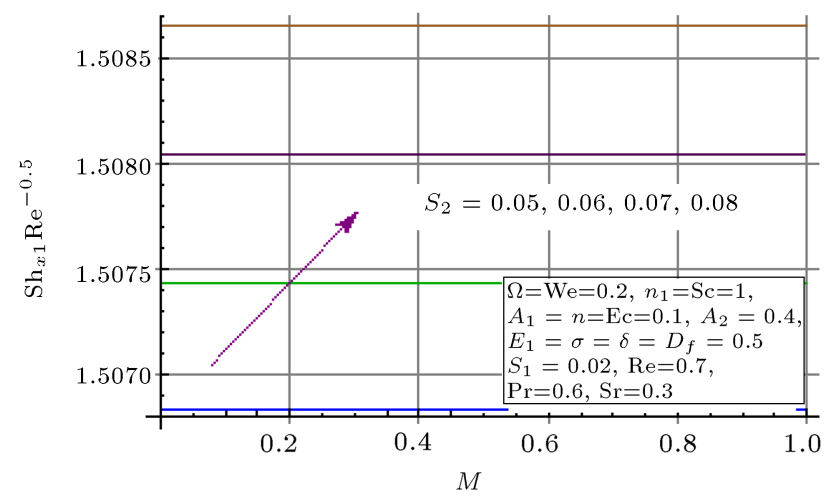

Figure 34. Sherwood number via $S_{2}$.

\section{Conclusions}

The following observations are worth mentioning:

- Velocities $\hat{h}(\xi), \hat{f}(\xi)$ and $\hat{g}(\xi)$ were reduced for $M$ at both disks, while $\hat{h}(\xi)$ and $\hat{f}(\xi)$ were increasing functions of We;

- For larger $S_{1}$ and $\operatorname{Pr}$, the temperature $\hat{\theta}(\xi)$ was reduced, while We and $D_{1}$ experienced an ascending trend;

- Opposite trends of concentration $\hat{\varphi}(\xi)$ were found for $S_{2}$ and Sc;
- Increasing behavior of Re for the skin friction was observed;

- Entropy rate through $(\mathrm{Br}),(L)$, and (We) was enhanced, while it decreased for thermal and solutal stratification parameters $\left(S_{1}\right)$ and $\left(S_{2}\right)$;

- Bejan number showed a decreasing behavior for larger $\left(S_{1}\right),\left(S_{2}\right)$, (We), and $(\mathrm{Br})$, while it increased for larger $(L)$.

\section{Acknowledgement}

We are grateful to Higher Education Commission (HEC) of Pakistan for financial support of this work under the project number No. 203038/NRPU/R\&D/HEC/13.

\section{References}

1. Williamson, R.V. "The flow of pseudoplastic materials", Indust. Eng. Chem., 21, pp. 1108-1111 (1929).

2. Nadeem, S., Hussain, S.T., and Lee, C. "Flow of a Williamson fluid over a stretching sheet", Brazilian J. Chem. Eng., 30, pp. 619-625 (2013).

3. Zehra I., Yousaf, M.M., and Nadeem, S. " Numerical solutions of Williamson fluid with pressure dependent viscosity", Results in Physics, 5, pp. 20-25 (2015).

4. Khan, M.I., Khan, T.A., Hayat, T., et al. "Irreversibility analysis and heat transfer performance of Williamson nanofluid over a stretched surface", Heat Transfer Research, 50(9) (2018). DOI: 10.1615/HeatTransRes.2018026342

5. Qayyum, S., Khan, M.I., Hayat, T., et al. "Entropy generation in dissipative flow of Williamson fluid between two rotating disks", Int. J. Heat Mass Transf., 127, pp. 933-942 (2018).

6. Hsiao, K. "To promote radiation electrical MHD activation energy thermal extrusion manufacturing system efficiency by using Carreau-nanofluid with parameters control method", Energy, 130, pp. 486-499 (2017).

7. Hsiao, K. "Combined electrical MHD heat transfer thermal extrusion system using Maxwell fluid with radiative and viscous dissipation effects", Appl. Thermal Eng., 112, pp. 1281-1288 (2017).

8. Hsiao, K. "Micropolar nanofluid flow with MHD and viscous dissipation effects towards a stretching sheet with multimedia feature", Int. J. Heat Mass Transf., 112, pp. 983-990 (2017).

9. Hayat, T., Farooq, S., and Ahmad, B. "Impact of compliant walls on magneto hydrodynamics peristalsis of Jeffrey material in a curved configuration", Sci. Iranica, 25, pp. 741-750 (2018).

10. Hsiao, K. "Stagnation electrical MHD nanofluid mixed convection with slip boundary on a stretching sheet", Appl. Thermal Eng., 98, pp. 850-861 (2016). 
11. Kármán, T.V. "Über laminare and turbulente Reibung", ZAMM-Journal of Applied Mathematics and Mechanics/Zeitschrift für Angewandte Mathematik und Mechanik, 1, pp. 233-252 (1921).

12. Hayat, T., Qayyum, S., Imtiaz, M., and Alsaedi, A. "Radiative flow due to stretchable rotating disk with variable thickness", Results Phys., 7, pp. 156-165 (2017).

13. Khan, N.A., Aziz, S., and Khan, N.A. "MHD flow of Powell-Eyring fluid over a rotating disk", J. Taiwan Inst. Chem. Eng., 45, pp. 2859-2867 (2014).

14. Doh, D.H. and Muthtamilselvan, M. "Thermophoretic particle deposition on magnetohydrodynamic flow of micropolar fluid due to a rotating disk", Int. J. Mech. Sci., 130, pp. 350-359 (2017).

15. Khan, N.A., Aziz, S., and Khan, N.A. "Numerical simulation for the unsteady MHD flow and heat transfer of couple stress fluid over a rotating disk", Plos One, 9, e95423 (2014).

16. Griffiths, P.T., Stephen, S.O., Bassom, A.P., et al. "Stability of the boundary layer on a rotating disk for power-law fluids", J. Non-Newtonian Fluid Mech., 207, pp. 1-6 (2014).

17. Qayyum, S., Khan, M.I., Hayat, T., et al. "Entropy generation in dissipative flow of Williamson fluid between two rotating disks", Int. J. Heat Mass Transf., 127, pp. 933-942 (2018).

18. Bestman, A.R. "Natural convection boundary layer with suction and mass transfer in a porous medium", Int. J. Eng. Research, 14, pp. 389-396 (1990).

19. Makinde, O.D., Olanrewaju, P.O., and Charles, W.M. "Unsteady convection with chemical reaction and radiative heat transfer past a flat porous plate moving through a binary mixture", Afrika Matematika, 22, pp. 65-78 (2011).

20. Awad, F.G., Motsa, S., and Khumalo, M. "Heat and mass transfer flow in unsteady rotating fluid flow with binary chemical reaction and activation energy", Plos One, 9(9), e107622 (2014).

21. Shafique, Z., Mustafa, M., and Mushtaq, A. "Boundary layer flow of Maxwell fluid in rotating frame with binary chemical reaction and activation energy", Results Phys., 6, pp. 627-633 (2016).

22. Bejan, A. "A study of entropy generation in fundamental convective heat transfer", J. Heat Transf., 101 pp. 718-725 (1979).

23. Amani, E. and Nobari, M.R.H. "A numerical investigation of entropy generation in the entrance region of curved pipes at constant wall temperature", Energy, 36, pp. 4909-4918 (2011).

24. Hayat, T., Rafiq, M., Ahmad, B., and Asghar, S. "Entropy generation analysis for peristaltic flow of nanoparticles in a rotating frame", Int. J. of Heat Mass Transf., 108, pp. 1775-1786 (2017).
25. Shit, G.C., Haldar, R., and Mandal, S. "Entropy generation on MHD flow and convective heat transfer in a porous medium of exponentially stretching surface saturated by nanofluids", Adv. Powder Technol., 28, pp. 519-1530 (2017).

26. Afridi, M.I. and Qasim, M. "Entropy generation and heat transfer in boundary layer flow over a thin needle moving in a parallel stream in the presence of nonlinear Rosseland radiation", Int. J. Thermal Sci., 123, pp. 117-128 (2018).

27. Govindaraju, M., Ganesh, N.V., Ganga, B., et al. "Entropy generation analysis of magneto hydrodynamic flow of a nanofluid over a stretching sheet", J. Egyp. Math. Soci., 23, pp. 429-434 (2015).

28. Hayat, T., Nawaz, S., and Alsaedi, A. "Entropy generation in peristalsis with different shapes of nanomaterial", J. Mol. Liq., 248, pp. 447-458 (2017).

29. Hayat, T., Khan, M.I., Qayyum, S., et al. "Entropy generation in flow with silver and copper nanoparticles", Colloids Surfaces: A Physicochemical Eng. Aspects, 539, pp. 335-346 (2018).

30. Rezaie, N.Z., Darasi, S.R.D., Zarandi, M.H.F., et al. "Generalized heat transfer and entropy generation of stratified air-water flow in entrance of a mini-channel", Sci. Iranica, 24 pp. 2407-2417 (2017).

31. Liao, S.J., Homotopy Analysis Method in Nonlinear Differential Equations., Springer, Heidelberg, Germany (2012).

32. Hayat, T., Khan, M.I., Farooq, M., et al. "Stagnation point flow with Cattaneo-Christov heat flux and homogeneous-heterogeneous reactions", J. Mol. Liq., 220, pp. 49-55 (2016).

33. Sheikholeslami, M., Hatami, M., and Ganji, D.D. "Micropolar fluid flow and heat transfer in a permeable channel using analytical method", J. Mol. Liq., 194, pp. 30-36 (2014).

34. Hayat, T., Khan, M.I., Farooq, M., et al. "Impact of Cattaneo-Christov heat flux model in flow of variable thermal conductivity fluid over a variable thicked surface", Int. J. Heat Mass Transf., 99, pp. 702-710 (2016).

35. Sui, J., Zheng, L., Zhang, X., et al. "Mixed convection heat transfer in power law fluids over a moving conveyor along an inclined plate", Int. J. Heat Mass Transf., 85, pp. 1023-1033 (2015).

36. Hayat, T., Khan, M.I., Qayyum, S., et al. "Modern developments about statistical declaration and probable error for skin friction and Nusselt number with copper and silver nanoparticles", Chin. J. Phys., 55, pp. 2501-2513 (2017).

37. Turkyilmazoglu, M. "Convergence accelerating in the homotopy analysis method: A new approach", $A d v$. Appl. Math. Mech., 10(4), pp. 925-947 (2018). 
38. Hayat, T., Qayyum, S., Imtiaz, M., et al. "Impact of Cattaneo-Christov heat flux in Jeffrey fluid flow with homogeneous-heterogeneous reactions", Plos One, 11e, e0148662 (2016).

39. Hayat, T., Khan, M.I., Qayyum, S., et al. "Entropy generation in magneto hydrodynamic radiative flow due to rotating disk in presence of viscous dissipation and Joule heating", Phys. Fluids, 30, 017101 (2018).

40. Turkyilmazoglu, M. "Parametrized adomian decomposition method with optimum convergence", Trans. Modelling Comp. Simul., 27(4), pp. 1-22 (2017). DOI: $10.1145 / 3106373$

41. Turkyilmazoglu, M. "Determination of the correct range of physical parameters in the approximate analytical solutions of nonlinear equations using the adomian decomposition method", Mediterranean $J$. Mathem., 13, pp. 4019-4037 (2016).

42. Khan, M.I., Qayyum, S., Hayat, T., et al. "Entropy generation minimization and statistical declaration with probable error for skin friction coefficient and Nusselt number", Chin. J. Phys., 56, pp. 1525-1546 (2018).

\section{Biographies}

Tasawar Hayat is a Pakistani Mathematician who has made pioneering research contributions to the area of mathematical fluid mechanics. He is considered one of the leading mathematicians working in Pakistan and, currently, is a Professor of Mathematics at the Quaidi-Azam University. He has 2000 plus publications.

Faria Masood is an MPhil student at Quaid-I-Azam University. She is doing MPhil under the kind supervision of Prof. Dr. Tasawar Hayat.

Sumaira Qayyum is a Pakistani Mathematician who has made research contributions to the area of mathematical fluid mechanics. She is currently doing PhD under the supervision of Prof. Dr. Tasawar Hayat. She has many research publications in the field of fluid mechanics.

Muhammad Ijaz Khan is a Pakistani Mathematician who has made research contributions to the area of mathematical fluid mechanics. He is currently doing PhD under the supervision of Prof. Dr. Tasawar Hayat. He has 200 plus research publications in the field of Fluid Mechanics.

Ahmed Alsaedi is a Saudian mathematician who has made pioneering research contributions to the area of mathematical fluid mechanics. $\mathrm{He}$ is currently a Professor of Mathematics at the King Abdulaziz University, Saudi Arabia. 